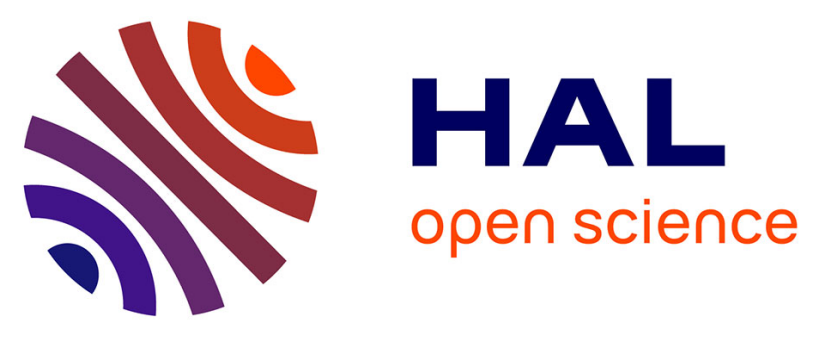

\title{
Sample Average Approximation for Multi-Vehicle Collection-Disassembly Problem under Uncertainty
}

Muhammad Khoirul Khakim Habibi, Olga Battaïa, Van-Dat Cung, Alexandre Dolgui, Manoj Kumar Tiwari

\section{- To cite this version:}

Muhammad Khoirul Khakim Habibi, Olga Battaïa, Van-Dat Cung, Alexandre Dolgui, Manoj Kumar Tiwari. Sample Average Approximation for Multi-Vehicle Collection-Disassembly Problem under Uncertainty. International Journal of Production Research, 2019, 57 (8), pp.2409-2428. 10.1080/00207543.2018.1519262 . hal-02434343

\section{HAL Id: hal-02434343 \\ https://hal.science/hal-02434343}

Submitted on 9 Jan 2020

HAL is a multi-disciplinary open access archive for the deposit and dissemination of scientific research documents, whether they are published or not. The documents may come from teaching and research institutions in France or abroad, or from public or private research centers.
L'archive ouverte pluridisciplinaire HAL, est destinée au dépôt et à la diffusion de documents scientifiques de niveau recherche, publiés ou non, émanant des établissements d'enseignement et de recherche français ou étrangers, des laboratoires publics ou privés. 
To appear in the International Journal of Production Research

Vol. 00, No. 00, 00 Month 20XX, 1-20

\title{
Sample Average Approximation for Multi-Vehicle Collection-Disassembly Problem under Uncertainty
}

\author{
Muhammad Khoirul Khakim Habibi ${ }^{\mathrm{a} *}$, Olga Battaïa ${ }^{\mathrm{b}}$, Van-Dat Cung ${ }^{\mathrm{c}, \mathrm{d}}$, Alexandre Dolgui ${ }^{\mathrm{e}}$ and Manoj \\ Kumar Tiwarif \\ ${ }^{a}$ École Nationale Supérieure des Mines de Saint-Étienne, Lab. LIMOS, CNRS UMR6158, 42023 \\ Saint-Étienne Cedex 2, France; \\ ${ }^{b}$ ISAE-SUPAERO, Université de Toulouse, 10 Avenue Edouard Belin, 31400 Toulouse, France; \\ ${ }^{c}$ Univ. Grenoble Alpes, G-SCOP, F-38000 Grenoble, France; \\ ${ }^{d} C N R S, G-S C O P$, F-38000 Grenoble, France;

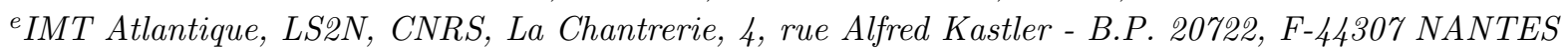 \\ Cedex 3, France; \\ ${ }^{f}$ Department of Industrial and Systems Engineering, Indian Institute of Technology Kharagpur, Kharagpur \\ 721 302, West Bengal, India
}

( $v 5.0$ released May 2017)

\begin{abstract}
The implementation of the circular economy is increasingly supported by many governments. It is performed by integrating the activities of reverse supply chain into those of forward supply chain. However, many companies that traditionally focus on the activities of forward supply chain have decided to collaborate with third-party reverse logistics providers to manage the reverse supply chain. This collaboration motivates the work presented in this paper to propose better decisions for decision makers in the providers under the fact that integrating decisions of the collection of End-of-Life products and their disassembly process proposes a reverse supply chain with better performance. In this paper, an integrated problem concerning those decisions is presented and formalised. It also deals with the uncertainty of the quality and the quantity of products as well as the demands of the associated components. Two approximate methods are developed to provide the solutions.
\end{abstract}

Keywords: Collection; Disassembly; Reverse Supply Chain; Stochastic Programming; Two-Phase Iterative Heuristic; Sample Average Approximation

\section{Introduction}

Nowadays, the evolution of the economic framework employed by the companies from the linear economy towards the circular economy is increasingly supported by many governments. The linear economy is characterised by "take-make-dispose" pattern where the raw materials are transformed into the final products in order to fulfil the demands of the clients and are disposed once they reach the end of life cycle. Based on the report of World Economic Forum (Forum 2014), the linear economy is arriving its limits in due to (i) the growth of resource prices and supply disruptions, (ii) the price volatility of metals, foods and non-agriculture outputs, (iii) the difficulty of creating sufficient competitive advantage or differentiation, (iv) the unpredicted consequences of the improvement of energy and resource efficiency, (v) the deceleration of agriculture productivity followed, (vi) the increasing risk of global supply chain's supply security and safety and (vii) the difficulties of getting virgin resources (water, land and atmosphere). Therefore, the implementation of the circular

*Corresponding author. Email: muhammad.habibi@emse.fr 
economy is encouraged due to its advantages in terms of financial, social and environmental factors. This implementation is carried out by incorporating the activities of reverse supply chain (RSC) dealing with End-of-Life (EOL) products i.e. reusing, repairing, refurbishing and recycling, to those of forward supply chain in order to form a closed-loop (circular) supply chain. Xerox, Hewlett-Packard and Phillips are among success story regarding such implementation (Kumar and Putnam 2008; Pishvaee, Farahani, and Dullaert 2010; Alumur et al. 2012; Forum 2014).

However, many companies that traditionally focus on the activities of forward supply chain have decided to collaborate with other specialised companies to manage RSC such as La Fédération ENVIE (France) since it requires new facilities and activities that are typically costly and manual labour intensive. This fact motivates this work to propose better decisions for decision makers in such third-party reverse logistics providers (3PRLPs). In detail, this work has been put in place in the particular interest of the management of EOL products from the points of collection until the point of re-manufacturers and/or recyclers.

Habibi et al. (2017a b) proved that integrating the decisions of the collection and disassembly processes of RSC leads to optimise and enhance the performance of the RSC in terms of total cost and the demand satisfaction. However, this work focuses on the deterministic case in which that the quality and the quantity of the EOL products as well as the demands of their components are well defined and only single vehicle is considered.

Table 1.: Comparison of Habibi et al. (2017a b) and this paper

\begin{tabular}{c|c|c}
\hline CHARACTERISTIC & Habibi et al. $(\overline{\mathbf{2 0 1 7 a}} \cdot \overline{\mathbf{b}})$ & This Paper \\
\hline Uncertainty & No & Yes \\
\hline $\begin{array}{c}\text { Parameters } \\
\text { affected by } \\
\text { uncertainty }\end{array}$ & No & $\begin{array}{c}\text { Quantity of products returned } \\
\text { Quantity of components } \\
\text { Demand of component }\end{array}$ \\
\hline No. of Vehicle & Single & Multiple \\
\hline
\end{tabular}

Reverse Supply Chain (RSC) needs to consider the uncertainty notably when it deals with Endof-Life (EOL) products. Based on McGovern and Gupta (2011), such products are often returned with imperfect or modified condition such as missing parts, components are replaced with higher quality ones etc. The quantity returned and the demand of component to sell are also highly uncertain.

Employing stochastic programming is an effective way to deal with such uncertainties. Managers of an RSC dealing with EOL products may refer to our work. They are able to optimize the performance of an RSC by minimizing the total cost containing the expected cost emerged from the uncertainties.

In this paper, we extend the works of Habibi et al. (2017a b) in order to approach the field reality by taking into account the uncertainty of the quality and the quality of EOL products as well as the demands of their components. The quality of product is assumed equivalent to the quantity of its components after it is returned to collection centres. We also take into account the case of multi-vehicle since 3PRLPs often posses more than one vehicle. Figure 1 depicts the contribution of this paper compared to the previous work.

\section{Literature Review}

The problem in Habibi et al. (2017a b), called Collection-Disassembly Problem (CDP), is a version of Production-Distribution Problem (PDP) or Production-Routing Problem (PRP) in RSC. It integrates decisions of two well-known and hard combinatorial problems i.e. vehicle routing and lot-sizing, to deal with the collection of EOL products and their disassembly process, respectively. 


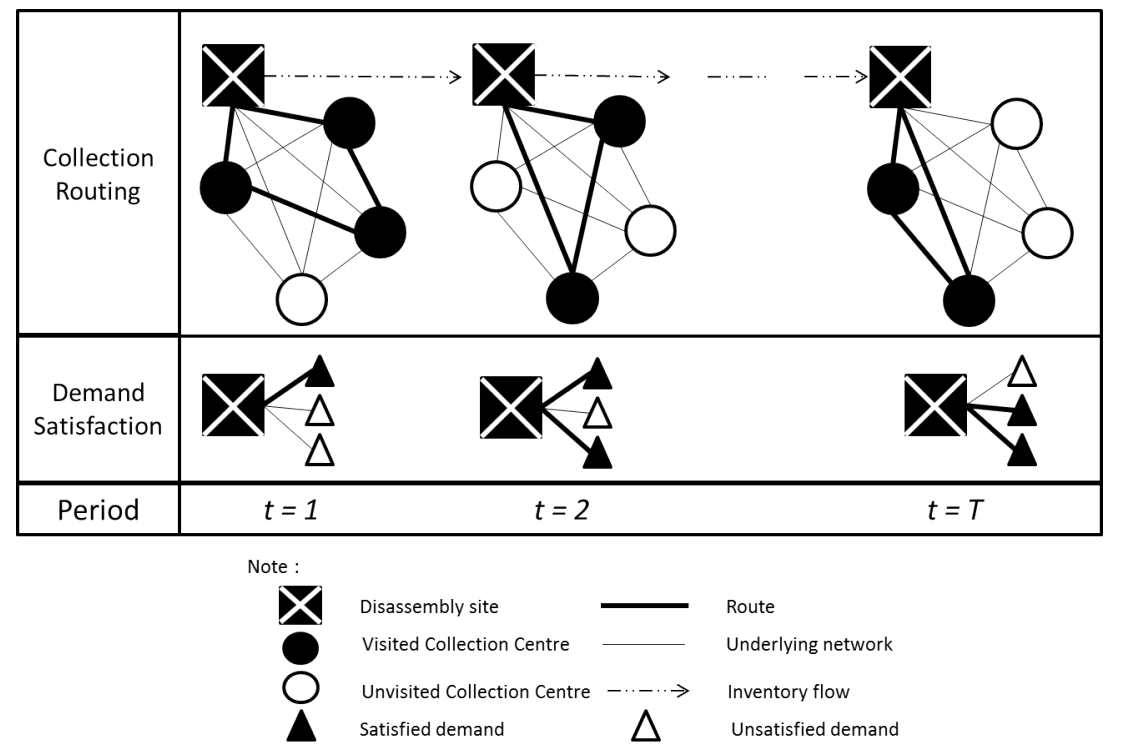

Figure 1.: Network Representations of Collection-Disassembly Problem Habibi et al. (2017a)

PDP differs from Newsvendor Problem. In their basic formulations, PDP concerns with multipleperiod plan whilst Newsvendor Problem considers single period. However, we are aware that Newsvendor Problem is advanced in multi-period. Newsvendor Problem considers no vehicle routing whilst it is an important part of PDP. Compared to routing problems, PDP takes into account decisions on production process. As an adaptation of PDP into RSC, the routing part of CDP concerns about the collection of End-of-Life products and the lot-sizing part lies in their disassembly process. In this work, we assume that some parameters are under uncertainty and more than one vehicle are available to use.

To well position our work in the existing literature, relevant papers in PDP are reviewed. The papers propose models and approximate methods particularly in dealing with large size instances when the commercial solver is not able to provide optimal solutions in acceptable CPU times.

The majority of PDP works focuses on both production and distribution aspects by incorporating the decisions of production and routing aspects in tactical level decision. Based on the existing literature of PDP that mostly deals with continuous products, the objective function minimises the total cost of production, inventory and routing, simultaneously, by respecting the demands of retailers, their inventory limits, the production facility's capacity and its inventory limit. As CDP, PDP is also a combination of two well-known and hard combinatorial problems i.e. lot-sizing problem and VRP, to deal with forward supply chain.

Based on Boudia, Louly, and Prins (2007); Bard and Nananukul (2009, 2010); Armentano, a.L. Shiguemoto, and Lø kketangen (2011), the decisions of PDP throughout the planning horizon consist of:

(1) when and how much products to produce

(2) when to visit and how much to deliver to retailers as well as the routing

(3) inventory level for each retailer and the depot

There are four existing models as the main references of PDP with multi-vehicle under Maximum Level policy. First, the formulation of Boudia, Louly, and Prins (2007) which is completed with vehicle index. Second, the formulation proposed in Bard and Nananukul (2009, 2010) has no index regarding the vehicle. Third, the formulation of Armentano, a.L. Shiguemoto, and Lø kketangen (2011) deals with the case of multi-products where the available vehicles are indexed. Fourth, the 
formulation of Adulyasak, Cordeau, and Jans (2015b) deals with PDP under the uncertainty of demands of retailers. Adulyasak, Cordeau, and Jans (2015a) and Díaz-Madroñero, Peidro, and Mula (2015) provide extensive reviews on PDP. Table 2 contains works on PDP highlighting on its variants as well as solving methods proposed.

Table 2.: Variants and Solving Methods of Production-Distribution Problem

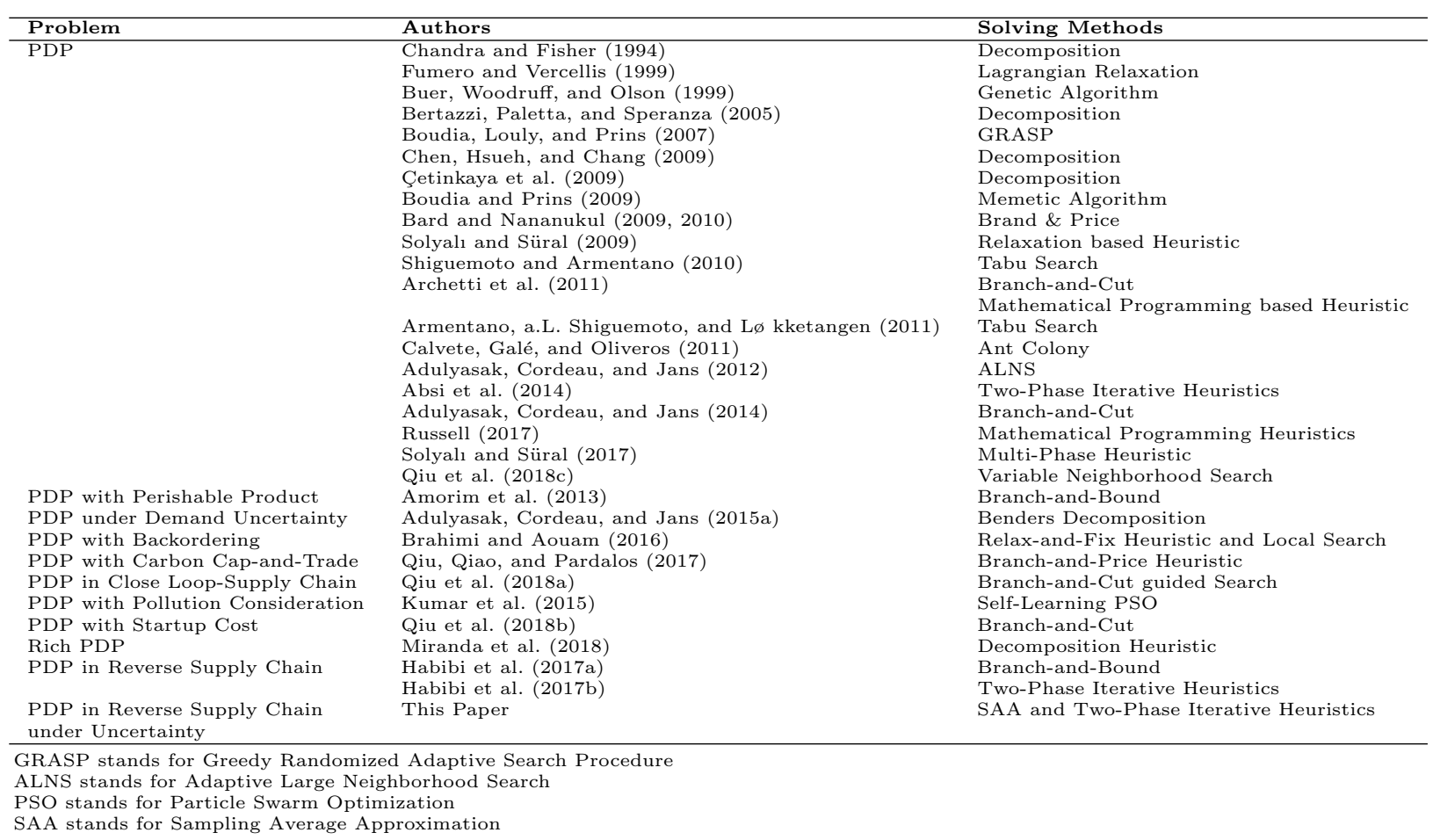

To the best of our knowledge, there is no work attempting to formalise the integration of decisions regarding the collection of EOL products and their disassembly process by taking into account the uncertainty of their quality and the quantity as well as the demands of their components. Also, there is no work proposing the case of multi-vehicle in such a problem. Therefore, a formulation filling this research gap is presented. Two methods are developed to provide the solutions to this problem.

\section{Problem Formulation}

This problem considers that a single site performs a disassembly process for treating a single type of EOL products available at dispersed collection centres. Some homogeneous vehicles with fixed capacity are available for collecting the EOL products.

The products' nomenclature is known and identical. Each product has several components where each component has uncertain quantity. The collected products are disassembled in the site in order to release the components requested. The site has a fixed capacity corresponding to its cycle time. A penalty cost is occured once the component demand is unmet. The demand of component is uncertain and assumed following some known distribution. The problem contains multi-period due to the presence of an inventory to store the collected EOL products. There is no salvage value or disposal cost for any leftover components. Following its characteristics, the problem is called as Stochastic Multi-Vehicle Collection-Disassembly Problem and abbreviated as SMCDP. In this work, the quality of product is assumed equivalent to the quantity of its components after it is returned to collection centers. We denote the quantity of each component a of the product at period 
$t$ under scenario $\omega$ as $n_{a t}^{\omega}$.

Parameters:

$\mathcal{A}$ set of component: $a=\{1,2, \cdots,|\mathcal{A}|\}$

$\mathcal{N}$ set of nodes: $i, j=\{1,2, \cdots,|\mathcal{N}|\}$ where 1 is the depot

$\mathcal{N}_{c} \quad$ set of collection centres: $i, j=\{2, \cdots,|\mathcal{N}|\}$

$\Omega \quad$ set of finite scenario: $\omega=\{1,2, \cdots,|\Omega|\}$

$\mathcal{T}$ planning horizon: $t=\{1,2, \cdots,|\mathcal{T}|\}$

$\mathcal{K} \quad$ set of vehicles: $k=1,2, \cdot|\mathcal{K}|$

$\rho^{\omega} \quad$ probability of scenario $\omega$

$n_{a t}^{\omega} \quad$ quantity of component $a$ in the product at period $t$ under scenario $\omega$

$S_{i t}^{\omega} \quad$ quantity of products available at collection centre $i$ at period $t$ under scenario $\omega$

$q_{a t}^{\omega} \quad$ demand of component $a$ at period $t$ under scenarion $\omega$

$Q \quad$ vehicle capacity

InvCap inventory capacity

DisCap disassembly line capacity imposed from its cycle time

$C F \quad$ fixed vehicle dispatch cost

$c_{i j} \quad$ mileage cost from node $i$ to $j$

$C D \quad$ unit disassembly cost

$\mathrm{CH} \quad$ unit holding cost

$C P_{a} \quad$ unit penalty cost of component $a$.

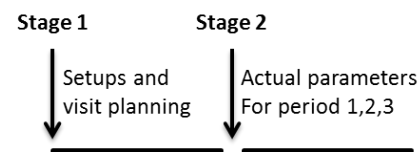

\begin{tabular}{ll|l|l|l|l|l|}
\hline Period 0 & Period 1 Period 2 \\
\hline
\end{tabular}

Figure 2.: Two-Stage Stochastic Problem

The problem is formalised as a two-stage stochastic programming. In this problem, a scenario $\omega$ in period $t$ is a realisation of $S_{i t}^{\omega}, n_{a t}^{\omega}$ and $q_{a t}^{\omega}$ into a fixed value coming after the planning stage as depicted in Figure2,

The first-stage decisions correspond to the planning of the routing of vehicle $k, \in K$ for each period as follows:

$z_{t} \quad$ number of vehicles dispatched at period $t$

$x_{i j t}^{k} \quad 1$ if node $j$ visited immediately after $i$ by vehicle $k$ at period $t .0$ otherwise.

Consequently, the second-stage decisions correspond to the load of vehicles and the disassembly decisions. These decisions will be taken after the realisation of the parameter under uncertainty. The decisions are:

$y_{i t}^{k \omega} \quad$ load of vehicle $k$ after visiting node $i$ at period $t$ in scenario $\omega$

$I_{t}^{\omega} \quad$ inventory level of EOL products at period $t$ in scenario $\omega$

$P_{t}^{\omega} \quad$ quantity of EOL products disassembled at period $t$ in scenario $\omega$

$S O_{a t}^{\omega} \quad$ unmet demands of component $a$ at period $t$ in scenario $\omega$

Stochastic Integer Linear Programming of SMCDP:

$$
\operatorname{Min} \sum_{t \in \mathcal{T}}\left(C F \cdot z_{t}+\sum_{k \in \mathcal{K}} \sum_{i, j \in \mathcal{N}} c_{i j} \cdot x_{i j t}^{k}+\sum_{\omega \in \Omega} \rho^{\omega}\left(C H \cdot I_{t}^{\omega}+C D \cdot P_{t}^{\omega}+\sum_{a \in \mathcal{A}} C P_{a} \cdot S O_{a t}^{\omega}\right)\right)
$$

subject to: 


$$
\begin{aligned}
& \sum_{k \in \mathcal{K}} \sum_{j \in \mathcal{N}, i \neq j} x_{i j t}^{k} \leq 1 \quad \forall i \in \mathcal{N}_{c}, \forall t \in \mathcal{T} \\
& \sum_{i \in \mathcal{N}_{c}} x_{1 i t}^{k} \leq 1 \quad \forall k \in \mathcal{K}, \forall t \in \mathcal{T} \\
& \sum_{k \in \mathcal{K}} \sum_{i \in \mathcal{N}_{c}} x_{1 i t}^{k} \leq z_{t} \quad \forall t \in \mathcal{T} \\
& \sum_{i \in \mathcal{N}, i \neq v} x_{i v t}^{k}=\sum_{j \in \mathcal{N}, j \neq v} x_{v j t}^{k} \quad \forall v \in \mathcal{N}, \forall k \in \mathcal{K}, \forall t \in \mathcal{T} \\
& y_{i t}^{k \omega}+\left(Q-S_{i t}^{\omega}\right) \cdot x_{1 i t}^{k} \leq Q \quad \forall i \in \mathcal{N}_{c}, \forall k \in \mathcal{K}, \forall t \in \mathcal{T}, \forall \omega \in \Omega \\
& y_{i t}^{k \omega}-y_{j t}^{k \omega}+Q \cdot x_{i j t}^{k}+\left(Q-S_{j t}^{\omega}-S_{i t}^{\omega}\right) \cdot x_{j i t}^{k} \leq Q-S_{j t}^{\omega} \quad i \neq j, \forall i, j \in \mathcal{N}_{c}, \forall k \in \mathcal{K}, \forall t \in \mathcal{T}, \forall \omega \in \Omega \\
& I_{t}^{\omega}=I_{t-1}^{\omega}+\sum_{k \in K} \sum_{i, j \in \mathcal{N}, i \neq j} S_{i t}^{\omega} \cdot x_{i j t}^{k}-P_{t}^{\omega} \quad \forall t \in \mathcal{T}, \forall \omega \in \Omega \\
& n_{a t}^{\omega} \cdot P_{t}^{\omega}+S O_{a t}^{\omega} \geq q_{a t}^{\omega} \quad \forall a \in \mathcal{A}, \forall t \in \mathcal{T}, \forall \omega \in \Omega \\
& \sum_{j \in \mathcal{N}, i \neq j} S_{i t}^{\omega} \cdot x_{i j t}^{k} \leq y_{i t}^{k \omega} \leq \sum_{j \in \mathcal{N}, i \neq j} Q \cdot x_{i j t}^{k} \quad \forall i \in \mathcal{N}, \forall k \in \mathcal{K}, \forall t \in \mathcal{T}, \forall \omega \in \Omega \\
& I_{t}^{\omega} \leq \text { InvCap } \\
& \forall t \in \mathcal{T}, \forall \omega \in \Omega \\
& P_{t}^{\omega} \leq \text { DisCap } \\
& \forall t \in \mathcal{T}, \forall \omega \in \Omega \\
& z_{t} \leq|\mathcal{K}| \\
& x_{i j t}^{k} \in\{0,1\} \\
& \forall t \in \mathcal{T} \\
& z_{t}, y_{i t}^{k \omega}, S O_{a t}^{\omega}, I_{t}^{\omega}, P_{t}^{\omega} \in \mathbb{Z}^{+} \\
& \forall i, j \in \mathcal{N}, \forall k \in \mathcal{K}, \forall t \in \mathcal{T} \\
& \forall a \in \mathcal{A}, \forall i \in \mathcal{N}, \forall k \in \mathcal{K}, \forall t \in \mathcal{T}, \forall \omega \in \Omega \text {. }
\end{aligned}
$$

The objective function (1) minimises the total cost of the first-stage decision and the expected cost corresponding to the second-stage decisions. The first and second terms correspond to the dispatch and mileage vehicle costs. The latter terms consist of the expected costs of inventory, disassembly and penalty.

Constraints (2) state that a collection centre is visited at most once by any vehicle at period $t$. Constraints (3) ensure that vehicle $k$ is dispatched at most once at period $t$. Constraints (4) determine number of vehicles dispatched at period $t$. The flows of visiting and leaving a node are conserved by constraints (5). The load of vehicle $k$ after visiting a collection centre $i$ in which it is the first node visited is ensured by constraints (6). Constraint (7) eliminate any subtour. Constraints (8) are the inventory balance of disassembly site. Constraints (9) impose the demand fulfilment. Constraints (10), (11), (12) and (13) are the limitation of load of vehicle, inventory level, disassembly and maximum number of vehicles, respectively. Constraints (14) and (15) define the decision variables.

\section{Solution Methods}

As far as our knowledge, Two-Phase Iterative Heuristics (Absi et al. 2014) provides high quality solutions in PDP. We also experience that this method with the type of Iterative Method with 
Multi-Travelling Salesman Problem (IM-MultiTSP) performs very good in CDP and is also flexible enough to be adapted. Therefore, it is implemented in SMCDP in the hope that it also proposes good performance. Apart from that, we also propose an enhanced version of this method since this enhancements lead to better performance than the original one.

An algorithmic framework is implemented combined with both methods to provide statistical lower and upper bounds since the proposed formulation is stochastic discrete optimisation problem with finite number of scenario.

\subsection{Two-Phase Iterative Heuristic}

In this part, the implementation of this method is demonstrated into PDP and SMCDP, consecutively.

\subsubsection{In PDP}

This method is originally proposed in Absi et al. (2014) for dealing with PDP with multi-vehicle and single type of product. It decomposes the problem into two subproblems and solved them iteratively. The two subproblems are the lot-sizing subproblem with approximate visiting costs and the routing subproblem.

The lot-sizing subproblem with approximate visiting costs, also called as the first phase, deals with the decisions of when and how much products to produce, when to visit retailers and how much products to retailers. Consecutively, this phase provides the set of retailers served in each period. Also, the vehicle capacity is already taken into account in this subproblem. Accordingly, the second phase (the routing subproblem) aims to construct the route of vehicle dispatched for each period corresponding to the set of retailers served.

The approximate visiting costs of a retailer are initialised by multiplying the go-return running costs and the distance between the retailer and the production facility. For those who are served in a particular period, a particular procedure is used to update their corresponding approximate visiting costs (see next part).

A diversification mechanism of the approximate visiting costs are required to permit the method exploring the unvisited solution space. It is simply done by multiplying the current value of the costs by the number of retailers visited throughout the planning horizon plus one. One is to avoid zero multiplication when no retailer is visited. The method is provided in Algorithm 1 .

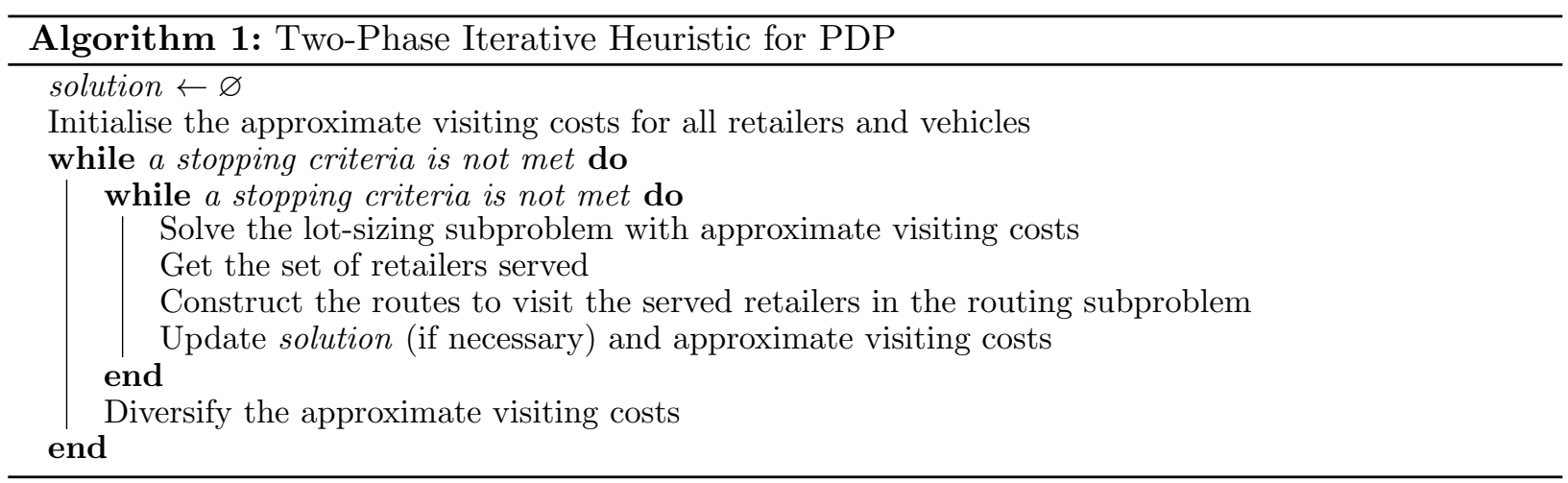

\subsubsection{In $S M C D P$}

This part consist of the adaptation of Two-Phase Iterative Heuristic for solving SMCDP. Some modifications are required due to the uncertainty of several parameters. 
The problem is decomposed into two subproblems: (i) Stochastic Multi-Vehicle Reverse LotSizing Problem with Approximate Visiting Costs (SMRLP-AVC) and (ii) Routing Problem.

4.1.2.1 SMRLP-AVC. This problem is a simplification of SMCDP by replacing the travelling cost with so-called approximated visiting cost. It determines how many vehicles to use $z_{t}$, which collection centre to visit, how many EOL products to store into the inventory $I_{t}^{\omega}$, how many EOL products to disassemble $P_{t}^{\omega}$ and how many penalty occurred $S O_{a t}^{\omega}$. Instead of using $c_{i j}$, it uses the approximate visiting costs denoted as $S C_{i t}^{k}$. These costs are initialised using $c_{0 i}+c_{i 0}$ and updated throughout the method.

The decision variables of SMRLP-AVC are described as follows:

$\gamma_{i t}^{k} \begin{cases}1 & \text { if node } i \text { is visited by vehicle } k \text { at period } t \\ 0 & \text { otherwise }\end{cases}$

$\beta_{t}^{k} \begin{cases}1 & \text { if vehicle } k \text { visits any collection centre at period } t \\ 0 & \text { otherwise }\end{cases}$

$r_{i t}^{k \omega} \quad$ quantity of EOL products collected from node $i$ by vehicle $k$ at period $t$ under scenario $\omega$

\subsubsection{Formulation of SMRLP-AVC.}

$\operatorname{Min} \sum_{t \in \mathcal{T}}\left(C F \cdot z_{t}+\sum_{k \in \mathcal{K}} \sum_{i \in \mathcal{N}_{c}} S C_{i t}^{k} \cdot \gamma_{i t}^{k}+\sum_{\omega \in \Omega} \rho^{\omega}\left(C H \cdot I_{t}^{\omega}+C D \cdot P_{t}^{\omega}+\sum_{a \in \mathcal{A}} C P_{a} \cdot S O_{a t}^{\omega}\right)\right)$

Subject to:

(9), 11), 12

$$
\begin{array}{ll}
I_{t}^{\omega}=I_{t-1}^{\omega}+\sum_{k \in \mathcal{K}} \sum_{i \in \mathcal{N}_{c}} r_{i t}^{k \omega}-P_{t}^{\omega} & \forall t \in \mathcal{T}, \forall \omega \in \Omega \\
r_{i t}^{k \omega}=S_{i t}^{\omega} \cdot \gamma_{i t}^{k} & \forall i \in \mathcal{N}_{c}, \forall k \in \mathcal{K}, \forall t \in \mathcal{T}, \forall \omega \in \Omega \\
\sum_{i \in \mathcal{N}_{c}} r_{i t}^{k \omega} \leq \min ^{k}\left\{Q ; \max _{a}\left\{\frac{\sum_{t^{\prime}=t}^{T} q_{a t^{\prime}}^{\omega}}{n_{a}}\right\}\right\} & \forall k \in \mathcal{K}, \forall t \in \mathcal{T}, \forall \omega \in \Omega \\
\sum_{k \in \mathcal{K}} \gamma_{i t}^{k} \leq 1 & \forall i \in \mathcal{N}_{c}, \forall t \in \mathcal{T}
\end{array}
$$

$$
\begin{array}{ll}
\sum_{i \in \mathcal{N}_{c}} \gamma_{i t}^{k} \leq\left|\mathcal{N}_{c}\right| \cdot \beta_{t}^{k} & \forall k \in \mathcal{K}, \forall t \in \mathcal{T} \\
\beta_{t}^{k} \leq \sum_{i \in \mathcal{N}_{c}} \gamma_{i t}^{k} & \forall k \in \mathcal{K}, \forall t \in \mathcal{T} \\
\sum_{k \in \mathcal{K}} \beta_{t}^{k}=z_{t} & \forall t \in \mathcal{T} \\
z_{t} \leq|\mathcal{K}| & \forall t \in \mathcal{T} \\
\gamma_{i t}^{k}, \beta_{t}^{k} \in\{0,1\} & \forall i \in \mathcal{N}_{c}, \forall k \in \mathcal{K}, \forall t \in \mathcal{T} \\
S O_{a t}^{\omega}, I_{t}^{\omega}, P_{t}^{\omega}, r_{i t}^{k \omega}, z_{t} \in \mathbb{Z}^{+} & \forall a \in \mathcal{A}, \forall i \in \mathcal{N}_{c}, \forall k \in \mathcal{K}, \forall t \in \mathcal{T}, \forall \omega \in \Omega .
\end{array}
$$

The objective function 16 is to minimise the total cost consisting of total fixed cost of vehicles 
deployed, total approximate cost and the expected cost of second-stage decisions of inventory, quantity of EOL products disassembled and unmet demand.

Constraints (17) impose the inventory balance of EOL products. Constraints (18) state all EOL products belong to collection centre $i$ have to be picked up once it is visited by any vehicle. Constraints $(19)$ is the maximum limit of $r_{i t}^{k \omega}$. Constraints 20 guarantee that a collection centre is visited at most once by any vehicle for each period. Constraints 21) state that $\beta_{t}^{k}$ is equal to 1 if vehicle $k$ visited at least one collection centre in period $t$. Otherwise, $\beta_{t}^{k}$ is equal to 0 as imposed by constraints (22). Constraints (23) and (24) state that number of vehicles used in each period is limited to the number of available vehicles. The nature of the decision variables on both stages are imposed in constraints (25) and (26).

Based on the values of $\gamma_{i t}^{k}$ obtained by solving SMRLP-AVC, the route of each vehicle is constructed. If necessary, the decision values and $S C_{i t}^{k}$ are updated based on the objective value. Apart from its initial values, the diversification mechanism of $S C_{i t}^{k}$ is employed in order to move to the other solution space. The algorithm 2 provides the implementation of the method to SMCDP.
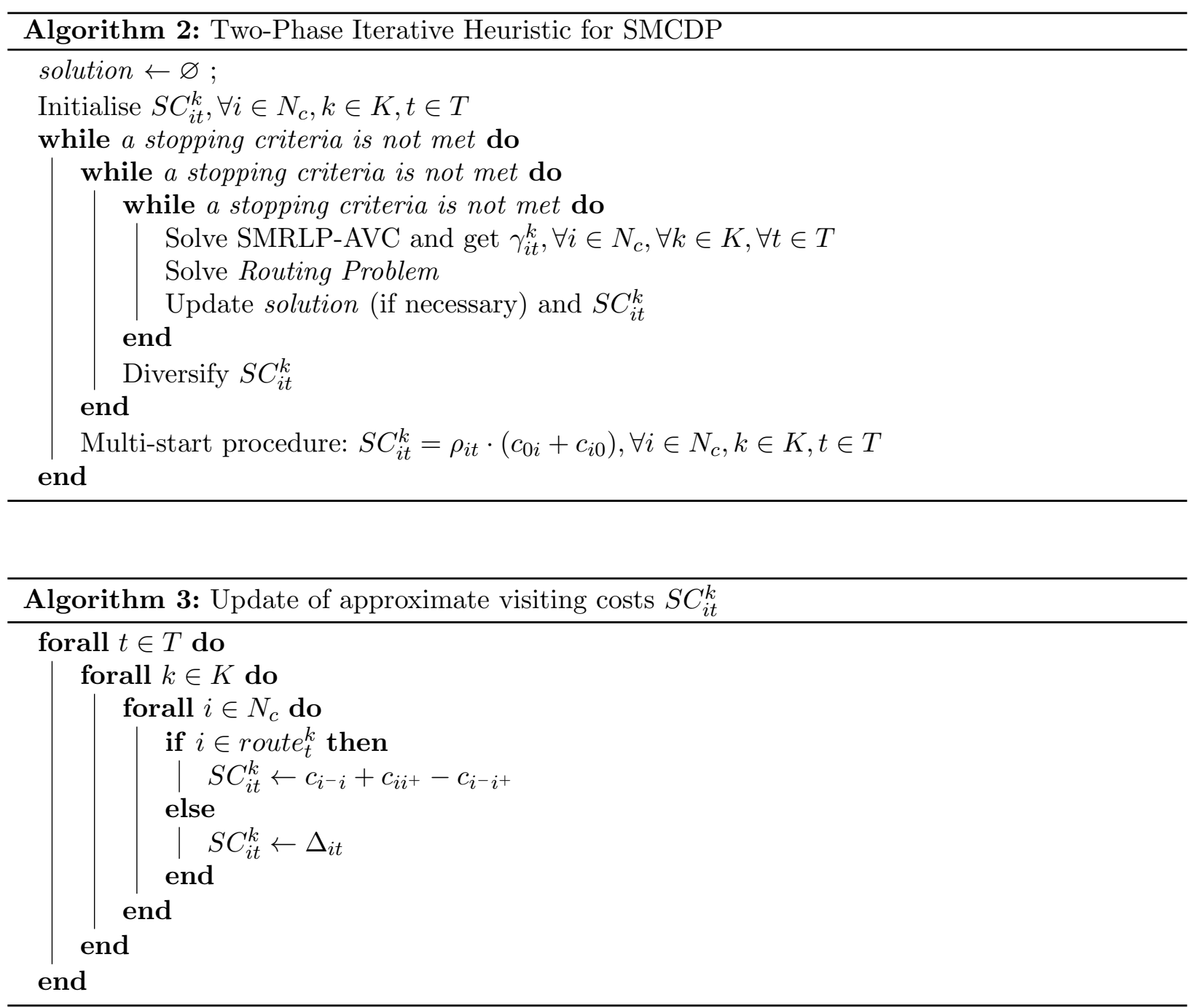

4.1.2.3 Routing Problem. The first phase provides the set of nodes served in each period. Thus, the routing problem becomes multi-TSP. To construct the route, we use the Lin-Kernighan Heuristic Lin and Kernighan (1973) as the the state-of-the-art heuristic of solving TSP. 


\subsubsection{Adaptive Two-Phase Iterative Heuristic}

In this part, the enhancement of Two-Phase Iterative Heuristic for SMCDP is described. It is proposed since it leads to faster computational time with good solutions for CDP.

In Algorithm 2, one notes that the problem is decomposed into SMRLP-AVC and routing problem. The solutions and $S C_{i t}^{k}$ are updated if the corresponding fitness value is better than previous one. In this enhancement, this step is denoted as the first step. The enhancement expands the method by putting the second and third additional steps in order to propose a better solution.

The second step introduces SMRLP-AVC II in order to provide the solution of SMRLP-AVC serving less periods by introducing the parameter $Z$ indicating number of periods served in SMRLPAVC. The variable $\alpha_{t}$ is equal to 1 if period $t$ is served. Otherwise, it is 0 . In this step, the approximate visiting costs $S C_{i t}^{k}$ of the second step are identical to the first step. SMRLP-AVC II is formalised as follows:

\subsubsection{SMRLP-AVC II.}

\section{Min 16}

Subject to:

$$
\begin{array}{ll}
(9),(11),(12), 17)-(26) & \\
z_{t} \leq|\mathcal{K}| \cdot \alpha_{t} & \forall t \in \mathcal{T} \\
\sum_{t \in \mathcal{T}} \alpha_{t} \leq Z-1 & \\
\alpha_{t} \in \mathbb{Z}^{+} & \forall t \in \mathcal{T} .
\end{array}
$$

Based on our experiences in the deterministic CDP, the second step of enhancements indeed provides better optimality gaps but longer CPU times than Two-Phase Iterative Heuristic. Therefore, an adaptive procedure is required to deal with this issue as follows.

This procedure is carried out by introducing the probability of using the second step denoted as prob. These value is halved once the step has no contribution to the solution by comparing it with a random values rand. This method is depicted in Algorithm 4.

The Algorithm 4 provides this enhancement as well as the adaptive procedure. The Algorithm 3 is also used in this enhanced method to update $S C_{i t}^{k}$.

\subsection{Sample Average Approximation}

Since SMCDP is stochastic discrete optimization problem, we adapt the Sample Average Approximation (SAA). This Monte Carlo-based sampling method is to tackle a problem having very large number of scenario denoted as $\Omega^{\prime}$, which is intractable, by solving the problem with a set of smaller and tractable scenario $\Omega$ where $|\Omega| \ll\left|\Omega^{\prime}\right|$ (Adulyasak, Cordeau, and Jans $2015 \mathrm{~b}$; Kleywegt, Shapiro, and Homem-de Mello 2002; Ghilas, Demir, and Woensel 2016).

The following is the procedure of SAA applied to our SMCDP:

1. Set replication $\mathcal{M}$ and generate scenario $\Omega$ as well as very large scenario $\Omega^{\prime}$ independently. The probability of each scenario $\omega$ associated with $|\Omega|$ is $\rho^{\omega}=\frac{1}{\Omega}$.

2. For $s=1 \rightarrow \mathcal{M}$, do :

2.1. Solve SMCDP. Store the objective value $Z_{\Omega}^{s}$, the vectors of the first stage solutions $\left(\mathbf{z}_{\Omega}^{s}, \mathbf{x}_{\Omega}^{s}\right)$ and the vectors of the second stage solutions $\left(\mathbf{I}_{\Omega}^{s}, \mathbf{P}_{\Omega}^{s}, \mathbf{S O}_{\Omega}^{s}\right)$. The average and 


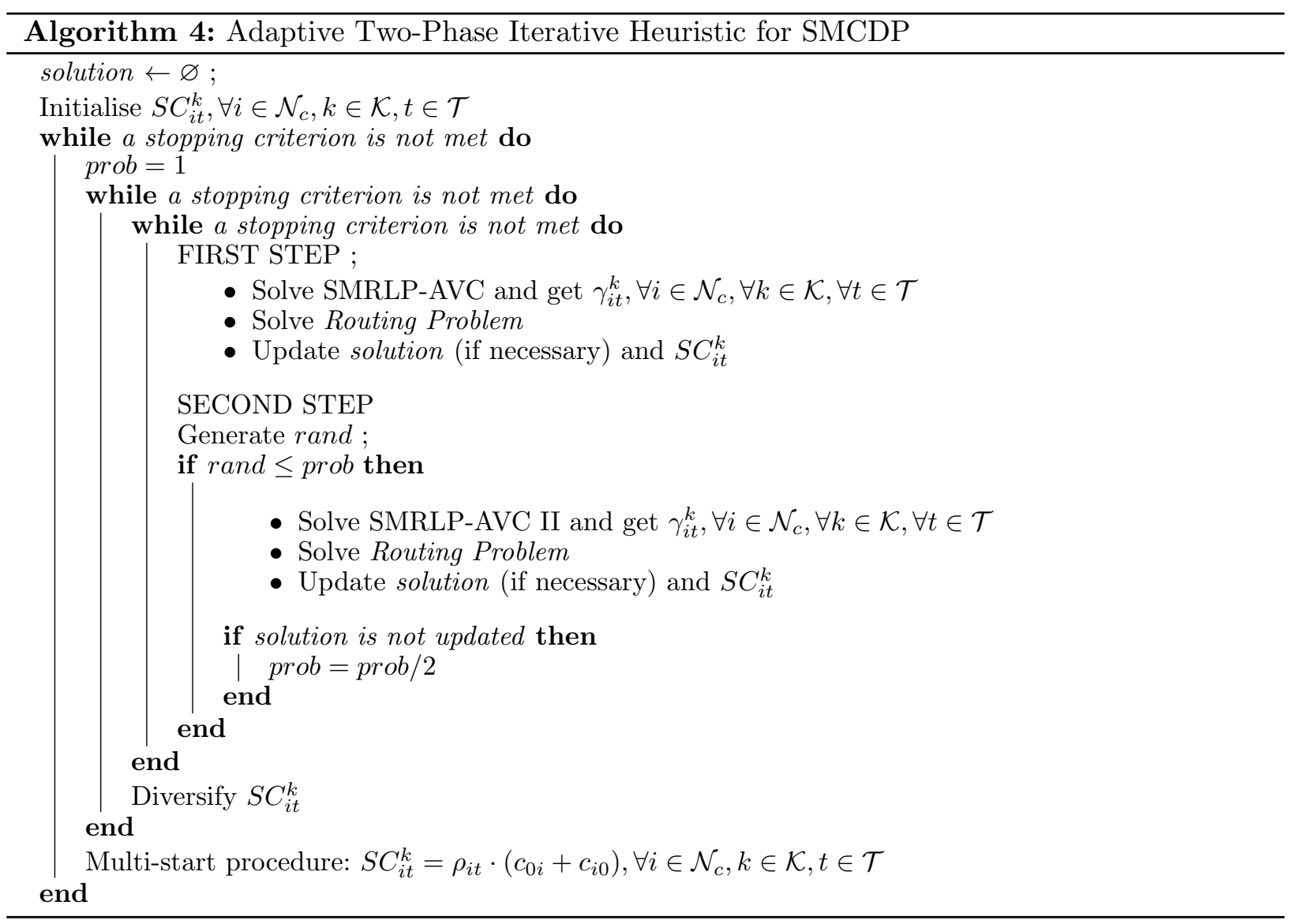

the variance of the objective value after $s$ replication denoted as $v_{\Omega}^{s}$ and $\sigma_{\Omega}^{s} 2$ are obtained as follows:

$$
\begin{aligned}
& v_{\Omega}^{s}=\frac{1}{s} \sum_{i=1}^{s} Z_{\Omega}^{i} \\
& \sigma_{\Omega}^{s} 2=\frac{1}{s \cdot(s-1)} \sum_{i=1}^{s}\left(Z_{\Omega}^{i}-v_{\Omega}^{s}\right)^{2}
\end{aligned}
$$

2.2. To obtain the second stage solutions $\mathbf{I}_{\Omega^{\prime}}^{s}, \mathbf{P}_{\Omega^{\prime}}^{s}$ and $\mathbf{S O}_{\Omega^{\prime}}^{s}$ of a very large scenario $\Omega^{\prime}$, use the best first stage solution after replication $s$ denoted as $\hat{\mathbf{z}}_{\Omega}^{s}$ and $\hat{\mathbf{x}}_{\Omega}^{s}$. The corresponding objective value (upper bound) is denoted as $v_{\Omega^{\prime}}\left(\hat{Z}^{s}\right)$ and its variance is obtained as follows:

$$
\sigma_{\Omega^{\prime}}^{2}=\frac{1}{\Omega^{\prime} \cdot\left(\Omega^{\prime}-1\right)} \sum_{\omega=1}^{\Omega^{\prime}}\left(G_{\omega}-v_{\Omega^{\prime}}\left(\hat{Z}^{s}\right)\right)^{2}
$$


where,

$$
G_{\omega}=\sum_{t \in T}\left\{C F \cdot \hat{z}_{t}+\sum_{k \in K} \sum_{i, j \in N} c_{i j} \cdot \hat{x}_{i j t}^{k}+C H \cdot \tilde{I}_{t}^{\omega}+C D \cdot \tilde{P}_{t}^{\omega}+\sum_{a \in A} C P_{a} \cdot \widetilde{S O}_{a t}^{\omega}\right\}
$$

Note that $\tilde{I}_{t}^{\omega}, \tilde{P}_{t}^{\omega}$ and $\widetilde{S O}_{a t}^{\omega}$ correspond to the second stage solution for scenario $\omega \in \Omega^{\prime}$. 2.3. Calculate the SAA gap $\varepsilon$ and its variance $\sigma_{\varepsilon}^{2}$ as follows:

$$
\begin{aligned}
& \varepsilon=v_{\Omega^{\prime}}\left(\hat{Z}^{s}\right)-v_{\Omega}^{s} \\
& \sigma_{\varepsilon}^{2}=\sigma_{\Omega^{\prime}}^{2}+\sigma_{\Omega}^{s}{ }^{2}
\end{aligned}
$$

3. Return $\hat{\mathbf{z}}_{\Omega}^{s}$ and $\hat{\mathbf{x}}_{\Omega}^{s}$ as the best solution.

\section{Numerical Experiments}

\section{$5.1 \quad$ Experimental Setup}

All formulations and algorithms were implemented in Java using Concert Technology and were solved by IBM CPLEX 12.6 on a PC with processor Intel@ Core ${ }^{\mathrm{TM}} \mathrm{i} 7 \mathrm{CPU} 2.9 \mathrm{GHz}$ and $4 \mathrm{~Gb}$ RAM under Windows 7 Professional.

The Monte Carlo simulation was used for scenario generation of the parameters associated with uncertainty $\left(S_{i t}^{\omega}, n_{a t}^{\omega}\right.$ and $\left.q_{a t}^{\omega}\right)$. They were generated independently by multiplying the corresponding values of deterministic CDP with random value following uniform distribution from 0 to 1.5 . The number of vehicles were set to 1,3 and 5 while the large scenario $\Omega^{\prime}$ were set to 1000 . To avoid memory issues, the maximum number of branch nodes of CPLEX for both two SMRLP-AVCs and large scenario problem of SAA is limited to 75000 . The methods were tested using instances 49,61 , 73, 85 and 97 of Data Sets of Random 1, Random 2, Cluster 1 and Cluster 2 presented in Habibi

\begin{tabular}{|c|c|c|c|c|c|}
\hline \multirow{2}{*}{ Instance } & \multicolumn{5}{|c|}{ Characteristic } \\
\hline & $|\mathcal{N}|$ & $|\mathcal{T}|$ & $|\mathcal{A}|$ & Demand & DisCap \\
\hline 49 & 10 & 10 & 10 & $U(40 \%: 60 \%) \cdot \boldsymbol{S}$ & $\infty$ \\
\hline 61 & 10 & 5 & 10 & $U(40 \%: 60 \%) \cdot \boldsymbol{S}$ & $\infty$ \\
\hline 73 & 5 & 25 & 10 & $U(40 \%: 60 \%) \cdot \boldsymbol{S}$ & $\infty$ \\
\hline 85 & 5 & 10 & 10 & $U(40 \%: 60 \%) \cdot \boldsymbol{S}$ & $\infty$ \\
\hline 97 & 5 & 10 & 5 & $U(40 \%: 60 \%) \cdot \boldsymbol{S}$ & $\infty$ \\
\hline
\end{tabular}
et al. (2017b). Their characteristic is provided in Table 3.

Table 3.: The Characteristic of Instances in All Data Sets

The three stopping criteria in Algorithms 2 and 4 are as follows:

(1) standard deviation of last ten fitness values, maximum iteration and CPU time are less than $5 \%, 100$ and 7200 seconds, respectively

(2) maximum number of diversification mechanism is 5

(3) maximum number of multi-start procedure is 5 . 


\subsection{Results}

For the sake of simplicity, $\mathcal{H}$ and $\mathcal{H}^{*}$ refer to Two-Phase Iterative Heuristic and Adaptive TwoPhase Iterative Heuristic, respectively. The summary of all solutions obtained is provided in Table 4. The details of all solutions are provided in Tables 7-9.

Table 4.: Results of All Data Sets

\begin{tabular}{|c|c|c|c|c|c|c|}
\hline \multirow{2}{*}{ Data Sets } & \multicolumn{3}{|c|}{$\mathcal{H}$} & \multicolumn{3}{|c|}{$\mathcal{H}^{*}$} \\
\hline & $\mu$ & $\bar{\sigma}$ & CPU Time & $\mu$ & $\sigma$ & CPU Time \\
\hline Random 1 & 539.53 & 26.90 & 1782.00 & 541.04 & 27.81 & 2030.58 \\
\hline Random 2 & 539.71 & 27.54 & 2162.05 & 540.76 & 27.87 & 2369.33 \\
\hline Cluster 1 & 539.27 & 27.61 & 1485.28 & 539.70 & 26.95 & 1423.26 \\
\hline Cluster 2 & 539.16 & 27.68 & 1438.43 & 540.13 & 26.73 & 1465.40 \\
\hline
\end{tabular}

According to Table 4, both methods provide solutions with no significant difference in terms of average and standard deviation. In terms of CPU times, $\mathcal{H}$ requires longer time in solving the data set of Random 1 rather than $\mathcal{H}^{*}$ as shown by Figures 3 and 4 .

Based on Figure 3, one notes that both methods are stable to solve the instances although there is a variation of scenario $\mathcal{M}$. However, the increase of the number of available vehicles $\mathcal{K}$ causes longer CPU times for both methods.

In order to further elaborate the results obtained, we conducted a sensitivity analysis on instance 61 of Random 2 data set. The analysis was conducted by varying the distribution of parameters under uncertainty and the multiplier value of increasing the penalty cost $C P_{a}$. We tested three types of distribution (Normal, Poisson and Uniform) and three values of penalty multiplication $(3,5$ and 10). For normal distribution, the mean and standard deviation of each parameter under uncertainty were set to the corresponding deterministic value in CDP and $50 \%$ of that value, respectively. For Poisson distribution, the only parameter was set to the corresponding deterministic value in CDP. For uniform distribution, it was set equally as mentioned in the previous section. We also define the small and large scenarios of SAA, $\Omega$ and $\Omega^{\prime}$, as 100 and 1100 , respectively.

In this regard, we add information regarding the two means for evaluating the stochastic solutions proposed by both solving methods called the Expected Value of Perfect Information (EVPI) and the Value of Stochastic Solutions (VSS). According to Birge and Louveaux (2011), EVPI and VSS measure the amount paid by a decision maker in return for a perfect information and is the cost incurred for ignoring the uncertainty, respectively. In practice, EVPI is the difference between the average objective value of solutions obtained for each scenario solved independently and the objective value of stochastic solution. Meanwhile, VSS is the difference between the objective value of stochastic solution and the objective value obtained using the average values of parameters under uncertainty. In our work, we obtain the two values based on SAA's very large set of scenarios $\Omega^{\prime}$.

Tables 5 and 6 depict the results obtained. EVPI and VSS are provided in \% against their corresponding objective function for SAA's very large set of scenario. One can see that the increase of penalty multiplier follows to the increase of SAA's average and standard deviation. It is also noticed that the value of EVPI (in \%) is following the increase of the penalty multiplier. Concerning the variation on the distribution type, the Poisson distribution proposes the lowest SAA's average and the Uniform distribution has the lowest EVPI (in \%).

Table 5.: Average Results on Penalty Multiplier Changes

\begin{tabular}{c|ccccc|ccccc}
\hline Penalty & \multicolumn{4}{|c|}{$\mathcal{H}$} & \multicolumn{3}{c}{$\mathcal{H}^{*}$} \\
\cline { 2 - 12 } Multiplier & $\mu$ & $\sigma$ & CPU Time & EVPI $\%$ & VSS $\%$ & $\mu$ & $\sigma$ & CPU Time & EVPI \% & VSS \% \\
\hline 3 & 1405.2 & 55.1 & 1315.9 & 54.1 & 3.1 & 1438.2 & 54.7 & 1921.3 & 53.7 & 4.1 \\
5 & 2779 & 63.7 & 1332.1 & 46.8 & 2.8 & 1376.6 & 72.9 & 1662.4 & 51.8 & 9 \\
10 & 6118 & 169 & 1257.9 & 40.5 & 1.4 & 6287.2 & 137 & 1828.6 & 40.8 & 1.4 \\
\hline
\end{tabular}


Table 6.: Average Results of Distribution Type Change

\begin{tabular}{c|ccccc|ccccc}
\hline \multirow{2}{*}{ Distribution } & \multicolumn{4}{|c}{$\mathcal{H}$} & \multicolumn{4}{c|}{$\mathcal{H}^{*}$} \\
\cline { 2 - 11 } & $\mu$ & $\sigma$ & CPU Time & EVPI $\%$ & VSS $\%$ & $\mu$ & $\sigma$ & CPU Time & EVPI \% & VSS \% \\
\hline Normal & 3715.1 & 250.3 & 1038.3 & 53.3 & 0 & 3820 & 221.3 & 1421.1 & 53.2 & 0 \\
Poisson & 3014.4 & 17.8 & 1515.1 & 49 & 3.7 & 1697.1 & 24.4 & 1975.1 & 54.2 & 10.6 \\
Uniform & 3572.7 & 19.7 & 1352.6 & 39.1 & 3.7 & 3584.8 & 18.9 & 2016.1 & 38.9 & 3.9 \\
\hline
\end{tabular}

3000,0
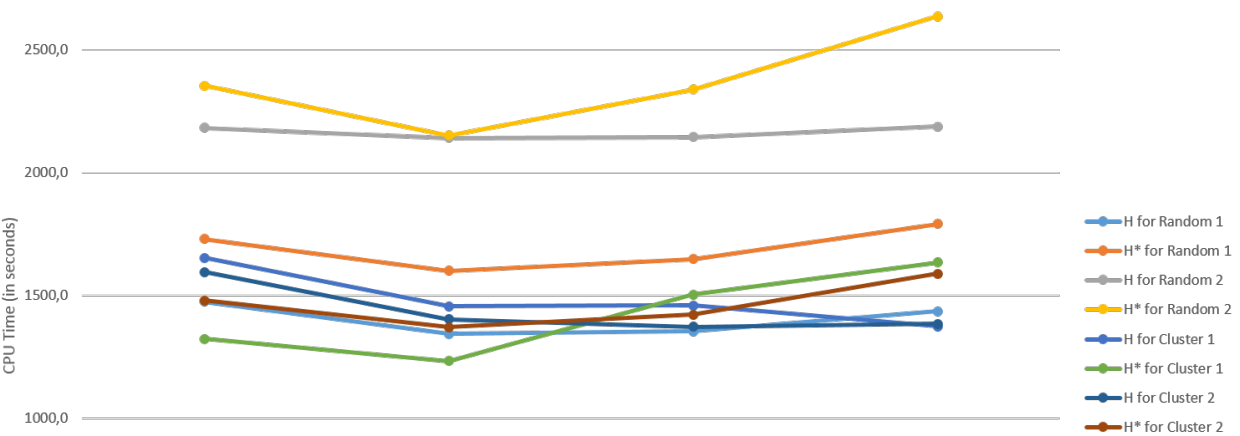

500,0

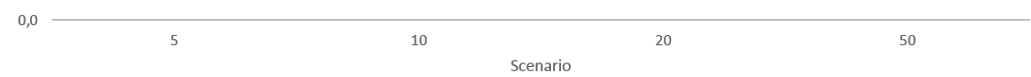

Figure 3.: Average CPU Times (in seconds) for All $\mathcal{M}$

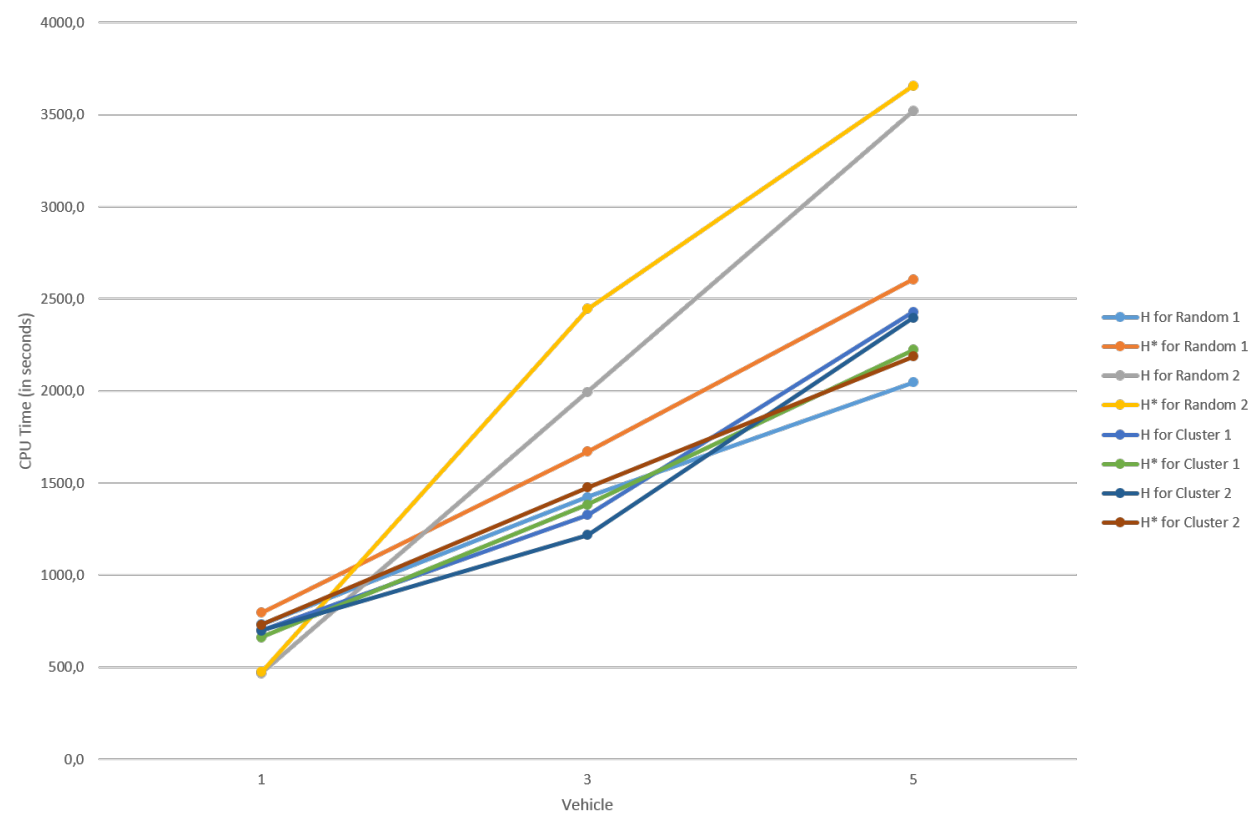

Figure 4.: Average CPU Times (in seconds) for All $\mathcal{K}$ 


\section{Conclusions and Future Works}

In this paper, the Stochastic Multi-Vehicle Collection-Disassembly Problem is formalised. It deals with the uncertainty of the quality and the quantity of EOL product as well as the demands of the associated components. The uncertain parameters are the number of components in each EOL product collected, the availability of EOL products at collection centres and their demands.

The problem is formalised as two-stage stochastic programming model in which the first-stage decisions have to be taken during the planning stage before any realisation of the uncertain parameters. The second-stage decisions are taken consecutively.

The first-stage decisions correspond to the number of vehicles dispatched and their routing decisions. Whilst, the second-stage decisions correspond to the inventory level, the number of disassembled and the unmet demands.

Accordingly, two approximate methods are developed to deal with i.e. Two-Phase Iterative Heuristic $(\mathcal{H})$ and Adaptive Two-Phase Iterative Heuristic $\left(\mathcal{H}^{*}\right) . \mathcal{H}$ decomposes the problem into two subproblems: the stochastic reverse lot-sizing problem with approximate visiting costs and the routing problem. Then, the two subproblems are solved iteratively. $\mathcal{H}^{*}$ is an enhanced version of $\mathcal{H}$ through additional steps and an adaptive procedure to improve the solution provided by both subproblems. In deterministic problem, $\mathcal{H}^{*}$ outperforms $\mathcal{H}$.

Both methods are combined with the algorithmic framework of Sample Average Approximation (SAA). This framework allow to solve a problem having very large scenario, which is intractable, by solving the problem with smaller and tractable scenario. Also, the scenario generation of each uncertain parameter is carried out using a Monte Carlo-based simulation.

Four data sets were tested in order to demonstrate the applicability of the methods to provide feasible solutions. A sensitivity analysis was conducted to evaluate the performance of both methods for different penalty costs and for various distribution types of parameters under uncertainty.

The realisation of the parameters under uncertainty in a such problem comes right after the planning stage. It is also possible that such realisation related to the second-stage decision variables might also occurs in each period following the multi-stage stochastic programming. To formalise the problem, the formulation of SMCDP can be extended with additional constraints related to the second-stage decisions e.g. the inventory level, products disassembled and unmet demands. These constraints ensure the consistency of the decisions between scenarios.

Furthermore, companies commonly deals with more than one type of products. It indicates that extending SMCDP by dealing with multi-products is highly possible. Consequently, additional index related to EOL products needs to be incorporated in term of formulation. Due to its flexibility and performance, the Two-Phase Iterative Heuristic of (Absi et al. 2014) combined with the rollout algorithm of Bertsekas, Tsitsiklis, and Wu (1997) may also be implemented to tackle such problem.

\section{Acknowledgement}

The government of Auvergne-Rhône-Alpes region of France is gratefully acknowledged for funding this work.

\section{References}

Absi, N, C Archetti, S Dauzère-Pérès, and D Feillet. 2014. "A two-phase iterative heuristic approach for the production routing problem." Transportation Science 49 (4): 784-795.

Adulyasak, Yossiri, Jean-François Cordeau, and Raf Jans. 2014. "Formulations and branch-and-cut algorithms for multivehicle production and inventory routing problems." INFORMS Journal on Computing 26 (1): 103-120. 
Table 7.: Results of Random 1

\begin{tabular}{|c|c|c|c|c|c|c|c|c|c|}
\hline \multirow{2}{*}{ Instance } & \multirow{2}{*}{$|\mathcal{K}|$} & \multirow{2}{*}{$|\Omega|$} & \multirow{2}{*}{$|\mathcal{M}|$} & \multicolumn{3}{|c|}{$\mathcal{H}$} & \multicolumn{3}{|c|}{$\mathcal{H}^{*}$} \\
\hline & & & & $\mu$ & $\sigma$ & CPU Time & $\mu$ & $\sigma$ & CPU Time \\
\hline \multirow[t]{12}{*}{49} & \multirow[t]{4}{*}{1} & 5 & 200 & 497.6 & 20.8 & 1094.5 & 493.5 & 20.3 & 1150.9 \\
\hline & & 10 & 100 & 487.4 & 20.2 & 847.7 & 500.8 & 19.6 & 1011 \\
\hline & & 20 & 50 & 480.1 & 20.3 & 804.8 & 493.4 & 21.9 & 1087.9 \\
\hline & & 50 & 20 & 486.3 & 24.2 & 753.3 & 500.3 & 20.6 & 818.4 \\
\hline & \multirow[t]{4}{*}{3} & 5 & 200 & 494.9 & 21.3 & 2266.1 & 495 & 22 & 2189.7 \\
\hline & & 10 & 100 & 494.4 & 20.7 & 2044.9 & 501 & 21.1 & 2033.4 \\
\hline & & 20 & 50 & 496.8 & 20.8 & 1846.7 & 491.1 & 19.3 & 1863.5 \\
\hline & & 50 & 20 & 496.2 & 28.2 & 1631.6 & 499.2 & 21.6 & 1915.6 \\
\hline & \multirow[t]{4}{*}{5} & 5 & 200 & 496.1 & 21.2 & 3165.3 & 501.6 & 22.3 & 3020.1 \\
\hline & & 10 & 100 & 491.4 & 21 & 2620.7 & 492.4 & 21.9 & 2740.7 \\
\hline & & 20 & 50 & 495 & 21.8 & 2787.3 & 500.3 & 23.3 & 2762.9 \\
\hline & & 50 & 20 & 501 & 19.9 & 3898.2 & 506.1 & 21.3 & 2741.1 \\
\hline \multirow[t]{12}{*}{61} & \multirow[t]{4}{*}{1} & 5 & 200 & 239.4 & 10.3 & 505.1 & 246.1 & 10 & 435.2 \\
\hline & & 10 & 100 & 239.5 & 10.3 & 380.7 & 247.3 & 9 & 378.8 \\
\hline & & 20 & 50 & 247.2 & 9.6 & 362.5 & 239.7 & 9.2 & 351.3 \\
\hline & & 50 & 20 & 241 & 11.5 & 333.8 & 242.1 & 8.9 & 283.8 \\
\hline & \multirow[t]{4}{*}{3} & 5 & 200 & 242.1 & 9.8 & 722.7 & 245.3 & 9.6 & 681.3 \\
\hline & & 10 & 100 & 240.1 & 9.3 & 686.1 & 241.9 & 10.2 & 589 \\
\hline & & 20 & 50 & 245.2 & 9.8 & 403.9 & 235.8 & 9.8 & 568 \\
\hline & & 50 & 20 & 236.5 & 9.1 & 500.7 & 245.5 & 13 & 485.2 \\
\hline & 5 & 5 & 200 & 241.8 & 10.5 & 869.3 & 244.7 & 9.5 & 779.5 \\
\hline & & 10 & 100 & 238.1 & 10.3 & 733.1 & 242 & 11 & 651.4 \\
\hline & & 20 & 50 & 242 & 9.6 & 636.4 & 241.3 & 9.3 & 596.2 \\
\hline & & 50 & 20 & 242 & 10.9 & 751.4 & 239.4 & 9.6 & 565.1 \\
\hline 73 & 1 & 5 & 200 & 1223.9 & 50.6 & 1882.6 & 1222.8 & 51.1 & 1767.2 \\
\hline & & 10 & 100 & 1233.4 & 48.1 & 1509.5 & 1210.2 & 55 & 1530.6 \\
\hline & & 20 & 50 & 1220.6 & 47.2 & 1568.4 & 1232 & 48 & 1503.8 \\
\hline & & 50 & 20 & 1217.4 & 49.1 & 1493.9 & 1234.2 & 40.7 & 1550.9 \\
\hline & 3 & 5 & 200 & 1227 & 51.4 & 3304.9 & 1225.2 & 49.3 & 3690.6 \\
\hline & & 10 & 100 & 1224.4 & 50.5 & 3470.4 & 1216.3 & 55 & 3962.8 \\
\hline & & 20 & 50 & 1219.4 & 42.3 & 3150.9 & 1218.8 & 57.5 & 4160.3 \\
\hline & & 50 & 20 & 1216.9 & 54.2 & 3578.6 & 1236.1 & 47 & 4497.5 \\
\hline & 5 & 5 & 200 & 1225.7 & 47.2 & 4285 & 1243.6 & 54.4 & 6365.8 \\
\hline & & 10 & 100 & 1225.3 & 50.2 & 4272 & 1221.9 & 48.9 & 6288.2 \\
\hline & & 20 & 50 & 1221.5 & 46.2 & 5372.5 & 1230.5 & 48.4 & 7675.5 \\
\hline & & 50 & 20 & 1226.3 & 41.6 & 5667.6 & 1224.5 & 67.5 & 9996.3 \\
\hline 85 & 1 & 5 & 200 & 498.8 & 20.3 & 566.2 & 501.4 & 21.7 & 784.4 \\
\hline & & 10 & 100 & 504.9 & 22.6 & 501.3 & 491.7 & 20.2 & 643.7 \\
\hline & & 20 & 50 & 499.4 & 20.1 & 489.5 & 488.8 & 20.9 & 519.2 \\
\hline & & 50 & 20 & 494.2 & 19.5 & 394.4 & 491.8 & 18.9 & 516.4 \\
\hline & 3 & 5 & 200 & 495.2 & 21.1 & 919.5 & 501.7 & 22.3 & 1468.9 \\
\hline & & 10 & 100 & 498.8 & 20.7 & 898.2 & 497 & 23.5 & 1237.7 \\
\hline & & 20 & 50 & 496.7 & 21.2 & 770.6 & 494.7 & 20.9 & 1038 \\
\hline & & 50 & 20 & 494.2 & 18.3 & 728.1 & 500.1 & 27.9 & 931.1 \\
\hline & 5 & 5 & 200 & 490.6 & 22.9 & 1184.6 & 498.6 & 20.8 & 1678.3 \\
\hline & & 10 & 100 & 492.9 & 21 & 1056.6 & 494 & 18 & 1413.2 \\
\hline & & 20 & 50 & 499.2 & 19.9 & 1027.3 & 495.5 & 21.2 & 1284.2 \\
\hline & & 50 & 20 & 497 & 21.3 & 886.6 & 495.2 & 20.1 & 1195.1 \\
\hline 97 & 1 & 5 & 200 & 243.7 & 9.5 & 340.3 & 242.5 & 10.1 & 432.4 \\
\hline & & 10 & 100 & 244.5 & 9.3 & 329.8 & 243.1 & 10.2 & 421 \\
\hline & & 20 & 50 & 239.3 & 11.9 & 248.2 & 241.1 & 10.6 & 338.7 \\
\hline & & 50 & 20 & 244.6 & 8.5 & 260.3 & 245.7 & 9.8 & 387.9 \\
\hline & 3 & 5 & 200 & 242.2 & 10 & 417.7 & 240.9 & 10.2 & 728.2 \\
\hline & & 10 & 100 & 242.1 & 10 & 391.4 & 245.1 & 10.9 & 536.9 \\
\hline & & 20 & 50 & 236.8 & 9.8 & 377.5 & 247.8 & 11 & 423.9 \\
\hline & & 50 & 20 & 240.8 & 10.7 & 370.2 & 243.4 & 10.4 & 440.4 \\
\hline & 5 & 5 & 200 & 242.2 & 9.6 & 598.1 & 241.6 & 9.6 & 763.5 \\
\hline & & 10 & 100 & 238 & 10.3 & 409.2 & 245.4 & 9.8 & 571.9 \\
\hline & & 20 & 50 & 241.9 & 9.2 & 455.5 & 246.4 & 9.4 & 542.2 \\
\hline & & 50 & 20 & 248.2 & 9.8 & 292.5 & 243.9 & 9 & 524.1 \\
\hline
\end{tabular}

$\mu$ is average

$\sigma$ is standard deviation

CPU time is in seconds

Adulyasak, Yossiri, Jean-François Cordeau, and Raf Jans. 2015a. "The production routing problem: A review of formulations and solution algorithms." Computers \&3 Operations Research 55: 141-152.

Adulyasak, Yossiri, Jean-François Cordeau, and Raf Jans. 2012. "Optimization-based adaptive large neighborhood search for the production routing problem." Transportation Science 48 (1): 20-45.

Adulyasak, Yossiri, JF Cordeau, and Raf Jans. 2015b. "Benders decomposition for production routing under demand uncertainty." Operations Research 63 (4): 851-867.

Alumur, Sibel a., Stefan Nickel, Francisco Saldanha-da Gama, and Vedat Verter. 2012. "Multi-period reverse logistics network design." European Journal of Operational Research 220 (1): 67-78.

Amorim, P., M.a.F. Belo-Filho, F.M.B. Toledo, C. Almeder, and B. Almada-Lobo. 2013. "Lot sizing versus batching in the production and distribution planning of perishable goods." International Journal of Production Economics 146 (1): 208-218.

Archetti, Claudia, Luca Bertazzi, Giuseppe Paletta, and M. Grazia Speranza. 2011. "Analysis of the maximum level policy in a production-distribution system." Computers $\& 3$ Operations Research 38 (12): 1731- 
Table 8.: Results of Random 2

\begin{tabular}{|c|c|c|c|c|c|c|c|c|c|}
\hline \multirow{2}{*}{ Instance } & \multirow{2}{*}{$|\mathcal{K}|$} & \multirow{2}{*}{$|\Omega|$} & \multirow{2}{*}{$|\mathcal{M}|$} & \multicolumn{3}{|c|}{$\mathcal{H}$} & \multicolumn{3}{|c|}{$\mathcal{H}^{*}$} \\
\hline & & & & $\mu$ & $\sigma$ & CPU Time & $\mu$ & $\bar{\sigma}$ & CPU Time \\
\hline \multirow[t]{12}{*}{49} & 1 & 5 & 200 & 493.5 & 21 & 1083.2 & 495.2 & 21 & 986.7 \\
\hline & & 20 & 50 & 491.2 & 23.8 & 1045.2 & 496.9 & 21.6 & 872.6 \\
\hline & & 5 & 200 & 493.5 & 21 & 1083.2 & 495.2 & 21 & 986.7 \\
\hline & & 50 & 20 & 495.4 & 20.4 & 835.8 & 498 & 22 & 781.6 \\
\hline & 3 & 10 & 100 & 498.2 & 21.3 & 1806.3 & 492.7 & 19.5 & 1678.9 \\
\hline & & 20 & 50 & 491.1 & 22.3 & 1647.2 & 505.4 & 19.9 & 1478.9 \\
\hline & & 5 & 200 & 490.6 & 20.8 & 2187.7 & 498.9 & 21.2 & 2161.6 \\
\hline & & 50 & 20 & 492 & 28.9 & 1929.7 & 496.3 & 23.4 & 1637.6 \\
\hline & 5 & 10 & 100 & 493.2 & 22.5 & 5976.1 & 502.4 & 21.5 & 2500 \\
\hline & & 20 & 50 & 492 & 24.8 & 6545.2 & 505.4 & 20 & 2482.6 \\
\hline & & 5 & 200 & 493.8 & 21.9 & 6001 & 493.1 & 21.2 & 2983.3 \\
\hline & & 50 & 20 & 497.6 & 21.2 & 4535.5 & 499.2 & 19.5 & 2642.6 \\
\hline \multirow[t]{12}{*}{61} & 1 & 10 & 100 & 243 & 9.1 & 278.5 & 242.9 & 10.4 & 307.2 \\
\hline & & 20 & 50 & 244.1 & 9.3 & 243.6 & 243.4 & 10.6 & 288.8 \\
\hline & & 5 & 200 & 247.3 & 9.5 & 341.1 & 241 & 10.1 & 309.5 \\
\hline & & 50 & 20 & 240.2 & 8.9 & 245.6 & 246.6 & 9 & 246.6 \\
\hline & 3 & 10 & 100 & 243.6 & 10.7 & 474.8 & 239.5 & 10.7 & 591.8 \\
\hline & & 20 & 50 & 237.8 & 10.8 & 484.2 & 241.8 & 9.6 & 532.2 \\
\hline & & 5 & 200 & 243.7 & 10.5 & 535.5 & 246.1 & 9.6 & 525.8 \\
\hline & & 50 & 20 & 237.8 & 9.5 & 376.4 & 242.4 & 10.8 & 398.9 \\
\hline & 5 & 10 & 100 & 242.9 & 10.3 & 644 & 237.5 & 9.3 & 675.3 \\
\hline & & 20 & 50 & 244.9 & 9.2 & 502.1 & 240 & 10 & 631.3 \\
\hline & & 5 & 200 & 249.3 & 9.7 & 708.7 & 244.2 & 9.2 & 779.5 \\
\hline & & 50 & 20 & 240.2 & 9.8 & 561.4 & 237.6 & 8.4 & 595.1 \\
\hline \multirow[t]{12}{*}{73} & 1 & 10 & 100 & 1225.5 & 47.7 & 2844.3 & 1223.4 & 50.2 & 2742.8 \\
\hline & & 20 & 50 & 1231 & 58.2 & 2422 & 1237 & 52.3 & 2533.2 \\
\hline & & 5 & 200 & 1209.4 & 53.1 & 2806 & 1231.8 & 54.8 & 3201.2 \\
\hline & & 50 & 20 & 1218.7 & 48.6 & 2403.6 & 1221.4 & 55.2 & 3031 \\
\hline & 3 & 10 & 100 & 1232.8 & 51 & 5773.8 & 1233.6 & 47 & 7329.7 \\
\hline & & 20 & 50 & 1220.2 & 47.7 & 6601.9 & 1228.6 & 57.1 & 8286.1 \\
\hline & & 5 & 200 & 1224.3 & 47.1 & 5757 & 1214 & 50.6 & 7632.9 \\
\hline & & 50 & 20 & 1218.7 & 46 & 6751.6 & 1221.4 & 54.5 & 10630.4 \\
\hline & 5 & 10 & 100 & 1227.7 & 49.9 & 8989 & 1238.6 & 50.2 & 11097.4 \\
\hline & & 20 & 50 & 1223.1 & 57.4 & 8883.3 & 1217.1 & 53.7 & 14068.7 \\
\hline & & 5 & 200 & 1226.1 & 50.3 & 8530 & 1221.7 & 53.2 & 11650.8 \\
\hline & & 50 & 20 & 1219.4 & 56.8 & 11522.3 & 1217.1 & 53.8 & 15947.8 \\
\hline \multirow[t]{12}{*}{85} & 1 & 10 & 100 & 497.7 & 23.6 & 619.7 & 499.3 & 21.1 & 658 \\
\hline & & 20 & 50 & 501.6 & 17.6 & 610.7 & 495.1 & 21.6 & 555.6 \\
\hline & & 5 & 200 & 501.9 & 19.7 & 770.4 & 492.6 & 19.3 & 819.7 \\
\hline & & 50 & 20 & 499.3 & 24.9 & 528.7 & 501.1 & 27.6 & 492.7 \\
\hline & 3 & 10 & 100 & 497.5 & 19.3 & 985.1 & 495.8 & 22.2 & 1271.1 \\
\hline & & 20 & 50 & 497.5 & 21.5 & 1021.5 & 494.1 & 20.3 & 943.7 \\
\hline & & 5 & 200 & 496.7 & 22.2 & 1199.6 & 497.7 & 20.9 & 1227 \\
\hline & & 50 & 20 & 491.7 & 24.3 & 846.6 & 492.7 & 24.1 & 827.5 \\
\hline & 5 & 10 & 100 & 490.4 & 21.5 & 1313 & 501.4 & 20.2 & 1296.2 \\
\hline & & 20 & 50 & 500 & 22.3 & 1173.1 & 496.9 & 18.2 & 1235.6 \\
\hline & & 5 & 200 & 502.1 & 20.3 & 1378 & 495 & 20.2 & 1708.2 \\
\hline & & 50 & 20 & 500.2 & 16.8 & 1159.8 & 489.5 & 20.4 & 1318.6 \\
\hline \multirow[t]{12}{*}{97} & 1 & 10 & 100 & 236.6 & 10.4 & 292.9 & 236.6 & 9.6 & 349.6 \\
\hline & & 20 & 50 & 240.5 & 9.1 & 272.8 & 241.5 & 9.3 & 297.6 \\
\hline & & 5 & 200 & 239.2 & 10 & 363.9 & 243.4 & 10 & 343.2 \\
\hline & & 50 & 20 & 242.2 & 8.7 & 276.6 & 243.6 & 10.1 & 276.6 \\
\hline & 3 & 10 & 100 & 248 & 9.9 & 399.3 & 239.8 & 10.2 & 448.1 \\
\hline & & 20 & 50 & 240.9 & 9.5 & 270.8 & 242.5 & 9.9 & 438.3 \\
\hline & & 5 & 200 & 236.3 & 9.3 & 444.5 & 242.5 & 10 & 510.7 \\
\hline & & 50 & 20 & 237.9 & 9.6 & 418 & 243.6 & 9.3 & 364.8 \\
\hline & 5 & 10 & 100 & 241.6 & 9.8 & 561.9 & 237.9 & 9.4 & 335.4 \\
\hline & & 20 & 50 & 246.2 & 11.3 & 424.4 & 239.1 & 10.2 & 425.3 \\
\hline & & 5 & 200 & 242.4 & 10.6 & 618.8 & 253.5 & 9.5 & 452.4 \\
\hline & & 50 & 20 & 240.9 & 11 & 416.3 & 242.8 & 10.3 & 336.4 \\
\hline
\end{tabular}

$\mu$ is average

$\sigma$ is standard deviation

CPU time is in seconds

1746 .

Armentano, V.a., a.L. Shiguemoto, and a. Lø kketangen. 2011. "Tabu search with path relinking for an integrated productiondistribution problem." Computers 83 Operations Research 38 (8): 1199-1209.

Bard, Jonathan F., and Narameth Nananukul. 2009. "Heuristics for a multiperiod inventory routing problem with production decisions." Computers $\&$ Industrial Engineering 57 (3): 713-723.

Bard, Jonathan F, and Narameth Nananukul. 2010. "Computers \& Operations Research A branch-andprice algorithm for an integrated production and inventory routing problem." Computers and Operation Research 37 (12): 2202-2217.

Bertazzi, Luca, G Paletta, and MG Speranza. 2005. "Minimizing the total cost in an integrated vendormanaged inventory system." Journal of heuristics 11 (5): 393-419.

Bertsekas, DP, JN Tsitsiklis, and Cynara Wu. 1997. "Rollout algorithms for combinatorial optimization." Journal of Heuristics 3 (3): 245-262.

Birge, John R, and Francois Louveaux. 2011. Introduction to stochastic programming. Springer Science \& 
Table 9.: Results of Cluster 1

\begin{tabular}{|c|c|c|c|c|c|c|c|c|c|}
\hline \multirow{2}{*}{ Instance } & \multirow{2}{*}{$|\mathcal{K}|$} & \multirow{2}{*}{$|\Omega|$} & \multirow{2}{*}{$|\mathcal{M}|$} & \multicolumn{3}{|c|}{$\mathcal{H}$} & \multicolumn{3}{|c|}{$\mathcal{H}^{*}$} \\
\hline & & & & $\mu$ & $\sigma$ & CPU Time & $\mu$ & $\sigma$ & CPU Time \\
\hline \multirow[t]{12}{*}{49} & \multirow[t]{4}{*}{1} & 10 & 100 & 493.2 & 22.1 & 790.5 & 496.1 & 19 & 614.3 \\
\hline & & 20 & 50 & 489.9 & 22.3 & 782.6 & 500 & 20.4 & 692 \\
\hline & & 5 & 200 & 489.7 & 19.8 & 1008.6 & 497.4 & 20.6 & 668.3 \\
\hline & & 50 & 20 & 503.9 & 17.7 & 725.3 & 497.2 & 20.6 & 706.1 \\
\hline & \multirow[t]{4}{*}{3} & 10 & 100 & 493.9 & 21.1 & 1288.5 & 487.7 & 20.4 & 1227 \\
\hline & & 20 & 50 & 500.7 & 18.4 & 1314.7 & 492.9 & 23.3 & 1207.5 \\
\hline & & 5 & 200 & 497.7 & 22 & 1528.8 & 499.5 & 20.2 & 1450.4 \\
\hline & & 50 & 20 & 489.1 & 16.9 & 1383 & 505.3 & 25.9 & 1214.8 \\
\hline & \multirow[t]{4}{*}{5} & 10 & 100 & 498 & 21 & 5431.5 & 496.7 & 19.7 & 1888.1 \\
\hline & & 20 & 50 & 501.4 & 19.5 & 4459.2 & 480.5 & 20.7 & 2099.5 \\
\hline & & 5 & 200 & 480.7 & 21.4 & 5917 & 484.7 & 21.3 & 2155.5 \\
\hline & & 50 & 20 & 489.9 & 22 & 2969.9 & 495.5 & 20.1 & 2245.9 \\
\hline \multirow[t]{12}{*}{61} & \multirow[t]{4}{*}{1} & 10 & 100 & 239.1 & 10.4 & 315.2 & 243 & 9.9 & 308.7 \\
\hline & & 20 & 50 & 240.5 & 9.5 & 329.9 & 239.4 & 10.7 & 256.3 \\
\hline & & 5 & 200 & 243.4 & 9.7 & 267.5 & 246.6 & 10.5 & 333.6 \\
\hline & & 50 & 20 & 244.3 & 10 & 400.2 & 247 & 10.2 & 309.3 \\
\hline & \multirow[t]{4}{*}{3} & 10 & 100 & 239.7 & 11.5 & 494.1 & 234.1 & 9.6 & 439.9 \\
\hline & & 20 & 50 & 241.2 & 11 & 463.4 & 243.6 & 9.2 & 410.7 \\
\hline & & 5 & 200 & 242.8 & 10.4 & 495.4 & 241.4 & 9.3 & 566.7 \\
\hline & & 50 & 20 & 237.6 & 9.6 & 364.2 & 241.3 & 11.5 & 440.7 \\
\hline & 5 & 10 & 100 & 241 & 10 & 803.8 & 236.4 & 10.7 & 701.1 \\
\hline & & 20 & 50 & 237.5 & 9 & 701.2 & 243 & 9 & 566.6 \\
\hline & & 5 & 200 & 241.7 & 10.1 & 895.1 & 241.7 & 9.9 & 680.5 \\
\hline & & 50 & 20 & 246.6 & 10.7 & 660.8 & 236.6 & 10.5 & 507.1 \\
\hline 73 & 1 & 10 & 100 & 1214.1 & 57.6 & 1681.3 & 1224.6 & 55.9 & 1432.6 \\
\hline & & 20 & 50 & 1207.3 & 47.7 & 1344.7 & 1229.2 & 50.4 & 1501.9 \\
\hline & & 5 & 200 & 1220.1 & 51.6 & 1740.5 & 1230.2 & 52.3 & 1602.8 \\
\hline & & 50 & 20 & 1236.6 & 61 & 1326 & 1220 & 43.8 & 1515.1 \\
\hline & 3 & 10 & 100 & 1229.2 & 55.3 & 3065.3 & 1224.2 & 47.7 & 3009.2 \\
\hline & & 20 & 50 & 1224.8 & 48.6 & 3516.6 & 1217.7 & 45 & 4726.1 \\
\hline & & 5 & 200 & 1223.1 & 50.8 & 3612.4 & 1222 & 49 & 3155.6 \\
\hline & & 50 & 20 & 1225.6 & 55.3 & 3870.4 & 1211.9 & 51.8 & 4366.4 \\
\hline & 5 & 10 & 100 & 1225 & 49.1 & 4431.5 & 1228.4 & 48.9 & 5279.9 \\
\hline & & 20 & 50 & 1224.1 & 53 & 5325.7 & 1222.2 & 51.5 & 7561 \\
\hline & & 5 & 200 & 1217.3 & 51.3 & 5139.9 & 1239.1 & 53 & 5021.3 \\
\hline & & 50 & 20 & 1231.1 & 49.5 & 5421.2 & 1217.4 & 52.4 & 9495.7 \\
\hline 85 & 1 & 10 & 100 & 494.6 & 20.2 & 513.2 & 496.4 & 22.5 & 538.3 \\
\hline & & 20 & 50 & 497.8 & 22.9 & 478.4 & 498 & 20.5 & 478.3 \\
\hline & & 5 & 200 & 493.9 & 23.2 & 614.6 & 495.8 & 20.6 & 547.2 \\
\hline & & 50 & 20 & 499.4 & 19.7 & 428.1 & 492.4 & 18.7 & 488.2 \\
\hline & 3 & 10 & 100 & 499.2 & 18.2 & 868.1 & 496.1 & 20.2 & 956.3 \\
\hline & & 20 & 50 & 493 & 18.3 & 891.9 & 500.2 & 23.3 & 987.1 \\
\hline & & 5 & 200 & 496.5 & 19.5 & 941.6 & 500 & 20.6 & 952.1 \\
\hline & & 50 & 20 & 497.3 & 19 & 809.7 & 488.1 & 22.3 & 949.2 \\
\hline & 5 & 10 & 100 & 498.7 & 19.3 & 992 & 496.9 & 21.3 & 984.5 \\
\hline & & 20 & 50 & 502.5 & 21.8 & 1086.4 & 491.9 & 18.9 & 1047.5 \\
\hline & & 5 & 200 & 492.4 & 20.4 & 1305.7 & 497.9 & 22.8 & 1288.5 \\
\hline & & 50 & 20 & 488 & 20.5 & 1074.4 & 500.6 & 23.1 & 1132.1 \\
\hline 97 & 1 & 10 & 100 & 237.7 & 10.5 & 310.3 & 242.4 & 10.4 & 334 \\
\hline & & 20 & 50 & 243.3 & 11.5 & 295.6 & 244.1 & 10.8 & 272.3 \\
\hline & & 5 & 200 & 241.5 & 10.2 & 350.2 & 243.6 & 9.2 & 355.2 \\
\hline & & 50 & 20 & 239.8 & 8.6 & 312.3 & 242.8 & 11.4 & 296.4 \\
\hline & 3 & 10 & 100 & 242.2 & 9.4 & 379.7 & 240.8 & 10.2 & 370 \\
\hline & & 20 & 50 & 245.2 & 9.2 & 385 & 243.1 & 10.7 & 413.7 \\
\hline & & 5 & 200 & 240.1 & 10.5 & 441.3 & 240.5 & 10.1 & 458.6 \\
\hline & & 50 & 20 & 243.6 & 8.9 & 399.2 & 246 & 10.2 & 357.7 \\
\hline & 5 & 10 & 100 & 239.3 & 9.2 & 479.1 & 241.5 & 10.1 & 414.1 \\
\hline & & 20 & 50 & 243.6 & 9.4 & 499.8 & 242 & 10.6 & 326.3 \\
\hline & & 5 & 200 & 244.9 & 10.1 & 525.7 & 243.9 & 9.6 & 610.9 \\
\hline & & 50 & 20 & 239.8 & 12.1 & 468.1 & 242 & 8.5 & 479.3 \\
\hline
\end{tabular}

$\mu$ is average

$\sigma$ is standard deviation

CPU time is in seconds

Business Media.

Boudia, M., M.a.O. Louly, and C. Prins. 2007. "A reactive GRASP and path relinking for a combined productiondistribution problem." Computers \& Operations Research 34 (11): 3402-3419.

Boudia, M., and C. Prins. 2009. "A memetic algorithm with dynamic population management for an integrated productiondistribution problem." European Journal of Operational Research 195 (3): 703-715.

Brahimi, Nadjib, and Tarik Aouam. 2016. "Multi-item production routing problem with backordering: a MILP approach." International Journal of Production Research 54 (4): 1076-1093.

Buer, MG Van, DL Woodruff, and RT Olson. 1999. "Solving the medium newspaper production/distribution problem." European Journal of Operational Research 115 (2): 237-253.

Calvete, Herminia I., Carmen Galé, and María-José Oliveros. 2011. "Bilevel model for productiondistribution planning solved by using ant colony optimization." Computers $\& 3$ Operations Research 38 (1): $320-327$.

Çetinkaya, Sla, Halit Üster, Gopalakrishnan Easwaran, and Burcu Baris Keskin. 2009. "An Integrated 
Table 10.: Results of Cluster 2

\begin{tabular}{|c|c|c|c|c|c|c|c|c|c|}
\hline \multirow{2}{*}{ Instance } & \multirow{2}{*}{$|\mathcal{K}|$} & \multirow{2}{*}{$|\Omega|$} & \multirow{2}{*}{$|\mathcal{M}|$} & \multicolumn{3}{|c|}{$\mathcal{H}$} & \multicolumn{3}{|c|}{$\mathcal{H}^{*}$} \\
\hline & & & & $\mu$ & $\sigma$ & CPU Time & $\mu$ & $\bar{\sigma}$ & CPU Time \\
\hline \multirow[t]{12}{*}{49} & 1 & 10 & 100 & 486.2 & 19.9 & 704.4 & 503.6 & 19.8 & 595 \\
\hline & & 20 & 50 & 497.8 & 20.3 & 747.3 & 490.5 & 22.6 & 613.4 \\
\hline & & 5 & 200 & 503.2 & 21 & 903.6 & 497.2 & 21.2 & 723.8 \\
\hline & & 50 & 20 & 502 & 23.5 & 705 & 492.4 & 20.1 & 637.3 \\
\hline & 3 & 10 & 100 & 500.2 & 20.4 & 1532.2 & 498.1 & 21.5 & 1512.9 \\
\hline & & 20 & 50 & 491.4 & 22.9 & 1273.8 & 497.6 & 18.9 & 1428.7 \\
\hline & & 5 & 200 & 488.3 & 20.8 & 1730.4 & 496.2 & 21 & 1829.5 \\
\hline & & 50 & 20 & 490.4 & 21 & 1360.1 & 491.7 & 20.3 & 1615 \\
\hline & 5 & 10 & 100 & 499 & 19.5 & 6153.6 & 497.9 & 23.1 & 2115.3 \\
\hline & & 20 & 50 & 497.8 & 22.1 & 4860 & 496.5 & 18.7 & 2017.9 \\
\hline & & 5 & 200 & 501 & 22.8 & 6611.2 & 497.5 & 21.4 & 2387.4 \\
\hline & & 50 & 20 & 502 & 26.9 & 3893.3 & 496.4 & 17.3 & 2377.4 \\
\hline \multirow[t]{12}{*}{61} & 1 & 10 & 100 & 239.8 & 10.1 & 362.1 & 247.2 & 9.5 & 375.1 \\
\hline & & 20 & 50 & 240.5 & 9.4 & 365.2 & 239.6 & 12.1 & 381.7 \\
\hline & & 5 & 200 & 241.8 & 10.8 & 485.6 & 238.2 & 10.1 & 414.6 \\
\hline & & 50 & 20 & 243.5 & 8.8 & 338.1 & 242.3 & 9.4 & 336.6 \\
\hline & 3 & 10 & 100 & 239.8 & 9.9 & 593.7 & 242.7 & 9.2 & 645.1 \\
\hline & & 20 & 50 & 233.7 & 9.5 & 576.5 & 241.9 & 9.3 & 482.1 \\
\hline & & 5 & 200 & 239.2 & 10.5 & 619.9 & 243 & 9.4 & 771.4 \\
\hline & & 50 & 20 & 240.4 & 8.5 & 531.9 & 245.8 & 10.2 & 621.5 \\
\hline & 5 & 10 & 100 & 244.5 & 10 & 741.8 & 242.6 & 10.3 & 738.5 \\
\hline & & 20 & 50 & 232.3 & 10.3 & 717.4 & 239.6 & 8.7 & 691.3 \\
\hline & & 5 & 200 & 241.1 & 11 & 915.1 & 242.5 & 9.9 & 924.2 \\
\hline & & 50 & 20 & 239.1 & 9.5 & 598.3 & 239.2 & 8.5 & 762.1 \\
\hline \multirow[t]{12}{*}{73} & 1 & 10 & 100 & 1215.6 & 53.2 & 1675.4 & 1223.9 & 53.2 & 1807.7 \\
\hline & & 20 & 50 & 1217.4 & 47.6 & 1422.6 & 1229.1 & 48 & 1590.1 \\
\hline & & 5 & 200 & 1218.5 & 50.7 & 1916.9 & 1222.5 & 50.1 & 2118.9 \\
\hline & & 50 & 20 & 1216.8 & 48.5 & 1665.1 & 1225.1 & 51.4 & 1652.7 \\
\hline & 3 & 10 & 100 & 1217.9 & 54 & 2626 & 1232.7 & 47.7 & 3798.4 \\
\hline & & 20 & 50 & 1223.7 & 52.2 & 3017.7 & 1224.2 & 51.8 & 3873.3 \\
\hline & & 5 & 200 & 1224.7 & 50.3 & 3494.5 & 1222.2 & 51.1 & 3673.4 \\
\hline & & 50 & 20 & 1230.7 & 57.8 & 3009.5 & 1226.1 & 41.6 & 4485.9 \\
\hline & 5 & 10 & 100 & 1230.6 & 48 & 3550 & 1214.7 & 50.9 & 5099.8 \\
\hline & & 20 & 50 & 1230 & 54 & 4700.4 & 1225.2 & 54.4 & 7049.2 \\
\hline & & 5 & 200 & 1227.7 & 53.1 & 3927.8 & 1219.2 & 50.1 & 5221.7 \\
\hline & & 50 & 20 & 1217.3 & 52.1 & 5893.3 & 1213.6 & 56.9 & 8144.8 \\
\hline \multirow[t]{12}{*}{85} & 1 & 10 & 100 & 490 & 23 & 531.8 & 494.7 & 21.8 & 550.9 \\
\hline & & 20 & 50 & 490 & 21.4 & 493.4 & 500.6 & 18.2 & 478.8 \\
\hline & & 5 & 200 & 495.4 & 21.3 & 494.8 & 494 & 19.8 & 628.3 \\
\hline & & 50 & 20 & 494.6 & 20.4 & 398.1 & 495.5 & 21.2 & 468.9 \\
\hline & 3 & 10 & 100 & 501.4 & 22.2 & 760.4 & 494.1 & 20 & 996.7 \\
\hline & & 20 & 50 & 493.1 & 22.8 & 665.2 & 496.1 & 17.6 & 782.9 \\
\hline & & 5 & 200 & 498.4 & 19.5 & 766.4 & 501.6 & 19.7 & 1035.1 \\
\hline & & 50 & 20 & 492.1 & 20.3 & 679.9 & 498.3 & 19 & 751.7 \\
\hline & 5 & 10 & 100 & 493.6 & 21.7 & 1047.8 & 492.4 & 18.8 & 1291.7 \\
\hline & & 20 & 50 & 491.1 & 20.8 & 948.3 & 493.9 & 20.9 & 1023.7 \\
\hline & & 5 & 200 & 489.9 & 21.3 & 1186.9 & 497.1 & 20.3 & 1337.8 \\
\hline & & 50 & 20 & 495.1 & 21.8 & 965.2 & 493.3 & 18.8 & 1044.3 \\
\hline \multirow[t]{12}{*}{97} & 1 & 10 & 100 & 246.4 & 9.9 & 177.5 & 239 & 10.7 & 319.7 \\
\hline & & 20 & 50 & 243.1 & 11.7 & 206.8 & 247.2 & 9.5 & 291.9 \\
\hline & & 5 & 200 & 245.8 & 11.7 & 234.8 & 241.4 & 9.7 & 360.1 \\
\hline & & 50 & 20 & 240 & 9 & 164.4 & 241 & 9.5 & 289.4 \\
\hline & 3 & 10 & 100 & 242.5 & 10.6 & 268.2 & 244.8 & 9.7 & 325.3 \\
\hline & & 20 & 50 & 243.6 & 9 & 261.5 & 244 & 9.8 & 273.4 \\
\hline & & 5 & 200 & 244.4 & 9.1 & 311.5 & 241.7 & 9.3 & 347.6 \\
\hline & & 50 & 20 & 236 & 12.3 & 271.5 & 239.2 & 9.6 & 286.9 \\
\hline & 5 & 10 & 100 & 244.5 & 9.6 & 322.8 & 242.2 & 9.5 & 392.5 \\
\hline & & 20 & 50 & 242.6 & 11.1 & 318.5 & 241.5 & 9.1 & 352.7 \\
\hline & & 5 & 200 & 242.8 & 10.5 & 324.1 & 246.7 & 10.3 & 418.5 \\
\hline & & 50 & 20 & 246.9 & 9.4 & 286.7 & 248.1 & 8.4 & 361.4 \\
\hline
\end{tabular}

$\mu$ is average

$\sigma$ is standard deviation

CPU time is in seconds

Outbound Logistics Model for Frito-Lay: Coordinating Aggregate-Level Production and Distribution Decisions." Interfaces 39 (5): 460-475.

Chandra, Pankaj, and ML Fisher. 1994. "Coordination of production and distribution planning." European Journal of Operational Research 72 (3): 503-517.

Chen, Huey-Kuo, Che-Fu Hsueh, and Mei-Shiang Chang. 2009. "Production scheduling and vehicle routing with time windows for perishable food products." Computers \& Operations Research 36 (7): 2311-2319.

Díaz-Madroñero, Manuel, David Peidro, and Josefa Mula. 2015. "A review of tactical optimization models for integrated production and transport routing planning decisions." Computers 83 Industrial Engineering

Forum, World Economic. 2014. Towards the circular economy: Accelerating the scale-up across global supply chains. Technical Report January. Geneva: World Economic Forum.

Fumero, F, and C Vercellis. 1999. "Synchronized development of production, inventory, and distribution schedules." Transportation science 33 (3): 330-340. 
Ghilas, Veaceslav, Emrah Demir, and Tom Van Woensel. 2016. "A scenario-based planning for the pickup and delivery problem with time windows, scheduled lines and stochastic demands." Transportation Research Part B: Methodological 91: 34-51.

Habibi, M.K. Khakim, Olga Battaïa, Van-Dat Cung, and Alexandre Dolgui. 2017a. "Collection-disassembly problem in reverse supply chain." International Journal of Production Economics 183: 334-344.

Habibi, MK Khakim, Olga Battaïa, Van-Dat Cung, and Alexandre Dolgui. 2017b. "An efficient two-phase iterative heuristic for Collection-Disassembly problem." Computers \& Industrial Engineering 110: 505514 .

Kleywegt, AJ, Alexander Shapiro, and T Homem-de Mello. 2002. "The sample average approximation method for stochastic discrete optimization." SIAM Journal on Optimization 12 (2): 479-502.

Kumar, Ravi Shankar, Karthik Kondapaneni, Vijaya Dixit, a. Goswami, L.S. Thakur, and M.K. Tiwari. 2015. "Multi-objective modeling of production and pollution routing problem with time window: A self-learning particle swarm optimization approach." Computers $\&$ Industrial Engineering .

Kumar, Sameer, and Valora Putnam. 2008. "Cradle to cradle: Reverse logistics strategies and opportunities across three industry sectors." International Journal of Production Economics 115 (2): 305-315.

Lin, S., and B. W. Kernighan. 1973. "An Effective Heuristic Algorithm for the Traveling-Salesman Problem." Operations Research 21 (2): 498-516.

McGovern, S, and SM Gupta. 2011. The disassembly line: balancing and modeling. 1st ed. New York: McGraw-Hill.

Miranda, Pedro L, Jean-François Cordeau, Deisemera Ferreira, Raf Jans, and Reinaldo Morabito. 2018. "A Decomposition Heuristic for a Rich Production Routing Problem." Computers \& Operations Research .

Pishvaee, Mir Saman, Reza Zanjirani Farahani, and Wout Dullaert. 2010. "A memetic algorithm for biobjective integrated forward/reverse logistics network design." Computers \&6 Operations Research 37 (6): 1100-1112.

Qiu, Yuzhuo, Ming Ni, Liang Wang, Qinqin Li, Xuanjing Fang, and Panos M Pardalos. 2018a. "Production routing problems with reverse logistics and remanufacturing." Transportation Research Part E: Logistics and Transportation Review 111: 87-100.

Qiu, Yuzhuo, Jun Qiao, and Panos M Pardalos. 2017. "A branch-and-price algorithm for production routing problems with carbon cap-and-trade." Omega 68: 49-61.

Qiu, Yuzhuo, Liang Wang, Xiaoling Xu, Xuanjing Fang, and Panos M Pardalos. 2018b. "Formulations and branch-and-cut algorithms for multi-product multi-vehicle production routing problems with startup cost." Expert Systems with Applications .

Qiu, Yuzhuo, Liang Wang, Xiaoling Xu, Xuanjing Fang, and Panos M Pardalos. 2018c. "A variable neighborhood search heuristic algorithm for production routing problems." Applied Soft Computing 66: 311-318.

Russell, Robert A. 2017. "Mathematical programming heuristics for the production routing problem." International Journal of Production Economics 193: 40-49.

Shiguemoto, André Luís, and Vinícius Amaral Armentano. 2010. "A tabu search procedure for coordinating production, inventory and distribution routing problems." International Transactions in Operational Research 17 (2): 179-195.

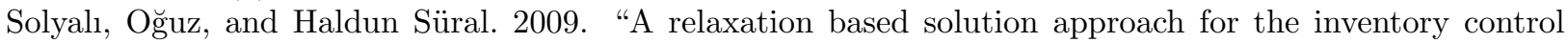
and vehicle routing problem in vendor managed systems." In Modeling, computation and Optimization, 171-189. World Scientific.

Solyalı, Ŏ̆uz, and Haldun Süral. 2017. "A multi-phase heuristic for the production routing problem." Computers \& Operations Research 87: 114-124. 